\title{
Mature myelin maintenance requires Qki to coactivate PPAR $\beta$-RXR $\alpha$-mediated lipid metabolism
}

\begin{abstract}
Xin Zhou, ${ }^{1}$ Chenxi He, ${ }^{2}$ Jiangong Ren, ${ }^{1}$ Congxin Dai, ${ }^{1,3}$ Sharon R. Stevens, ${ }^{4}$ Qianghu Wang, ${ }^{5}$ Daniel Zamler, ${ }^{6,7}$ Takashi Shingu, Liang Yuan, ${ }^{1,8}$ Chythra R. Chandregowda, ${ }^{1}$ Yunfei Wang, ${ }^{9}$ Visweswaran Ravikumar, ${ }^{10}$ Arvind U.K. Rao, ${ }^{10,11,12}$ Feng Zhou, ${ }^{13}$ Hongwu Zheng, ${ }^{14}$ Matthew N. Rasband, ${ }^{4}$ Yiwen Chen, ${ }^{15}$ Fei Lan, ${ }^{2}$ Amy B. Heimberger, ${ }^{16}$ Benjamin M. Segal,, ${ }^{17,18}$ and Jian Hu ${ }^{1,7}$

'Department of Cancer Biology, The University of Texas MD Anderson Cancer Center, Houston, Texas, USA. ²iver Cancer Institute, Zhongshan Hospital, Fudan University, Key Laboratory of Carcinogenesis and Cancer Invasion, Ministry of Education, and Key Laboratory of Epigenetics and Metabolism, Institutes of Biomedical Sciences, Fudan University, Shanghai, China. ${ }^{3}$ Department of Neurosurgery, Peking Union Medical College Hospital, Chinese Academy of Medical Sciences and Peking Union Medical College, Beijing, China. ㄹepartment of Neuroscience, Baylor College of Medicine, Houston, Texas, USA. ${ }^{5}$ Department of Bioinformatics, and Collaborative Innovation Center for Cancer Personalized Medicine, Nanjing Medical University, Nanjing, China. ${ }^{6}$ Department of Genomic Medicine, The University of Texas MD Anderson Cancer Center, Houston, Texas, USA. ${ }^{T}$ The University of Texas MD Anderson Cancer Center UTHealth Graduate School of Biomedical Sciences, Houston, Texas, USA. ${ }^{8}$ Craduate School of Biomedical Sciences, Tufts University, Boston, Massachusetts, USA. ${ }^{9}$ Department of Melanoma Medical Oncology, The University of Texas MD Anderson Cancer Center, Houston, Texas, USA. ${ }^{10}$ Department of Computational Medicine and Bioinformatics, University of Michigan, Ann Arbor, Michigan, USA. "Department of Radiation Oncology, University of Michigan, Ann Arbor, Michigan, USA. ${ }^{2}$ Department of Biomedical Engineering, University of Michigan, Ann Arbor, Michigan, USA. ${ }^{13}$ Liver Cancer Institute, Zhongshan Hospital, Fudan University, Key Laboratory of Carcinogenesis and Cancer Invasion, Ministry of Education, and Institutes of Biomedical Sciences, Shanghai, China. ${ }^{14}$ Department of Pathology and Laboratory Medicine, Weill Cornell Medicine, New York, USA. ${ }^{15}$ Department of Bioinformatics and Computational Biology, The University of Texas MD Anderson Cancer Center, Houston, Texas, USA. ${ }^{16}$ Department of Neurosurgery, The University of Texas MD Anderson Cancer Center, Houston, Texas, USA. ${ }^{17}$ Department of Neurology, The Ohio State University Wexner Medical Center, Columbus, Ohio, USA. ${ }^{18}$ The Neurological Research Institute, The Ohio State University, Columbus, Ohio, USA.
\end{abstract}

\begin{abstract}
Lipid-rich myelin forms electrically insulating, axon-wrapping multilayers that are essential for neural function, and mature myelin is traditionally considered metabolically inert. Surprisingly, we discovered that mature myelin lipids undergo rapid turnover, and quaking (Qki) is a major regulator of myelin lipid homeostasis. Oligodendrocyte-specific Qki depletion, without affecting oligodendrocyte survival, resulted in rapid demyelination, within 1 week, and gradually neurological deficits in adult mice. Myelin lipids, especially the monounsaturated fatty acids and very-long-chain fatty acids, were dramatically reduced by Qki depletion, whereas the major myelin proteins remained intact, and the demyelinating phenotypes of Qki-depleted mice were alleviated by a high-fat diet. Mechanistically, Qki serves as a coactivator of the PPAR $\beta-R X R \alpha$ complex, which controls the transcription of lipid-metabolism genes, particularly those involved in fatty acid desaturation and elongation. Treatment of Qki-depleted mice with PPARß/RXR agonists significantly alleviated neurological disability and extended survival durations. Furthermore, a subset of lesions from patients with primary progressive multiple sclerosis were characterized by preferential reductions in myelin lipid contents, activities of various lipid metabolism pathways, and expression level of QKI-5 in human oligodendrocytes. Together, our results demonstrate that continuous lipid synthesis is indispensable for mature myelin maintenance and highlight an underappreciated role of lipid metabolism in demyelinating diseases.
\end{abstract}

\section{Introduction}

Myelin is a specialized multilayer membrane structure that enables rapid saltatory nerve conduction, and it is assembled by oligodendrocytes in the central nervous system (CNS) (1). To sustain the normal function of neurons, the compact myelin structure needs to persist throughout adult life (1). Many myelinrelated CNS diseases, including multiple sclerosis (MS), develop in adulthood, underscoring the importance of active maintenance of the structural integrity of myelin in preventing neurological disability (2). Traditionally, demyelinating processes are thought to be the result of oligodendrocyte death induced by

Conflict of interest: The authors have declared that no conflict of interest exists. Copyright: () 2020, American Society for Clinical Investigation.

Submitted: July 14, 2019; Accepted: January 17, 2020; Published: March 23, 2020.

Reference information: / Clin Invest. 2020;130(5):2220-2236.

https://doi.org/10.1172/JCl131800. either extrinsic or intrinsic insults (3-5). However, evidence suggests that oligodendrocyte death is not the underlying etiology for all pathological demyelinating processes. More specifically, oligodendrocytes are largely preserved in 30\%-60\% of early active MS lesions with severe demyelination (6-8), and the turnover rates of oligodendrocytes remain generally low, albeit heterogeneous, across multiple CNS regions during aging despite global myelin volume decline (9-12). These observations suggest that myelin content could be tightly regulated in a manner that is independent of oligodendrocyte survival.

The dry mass of CNS myelin is composed of approximately $70 \%$ lipids and $30 \%$ proteins - a composition that is characterized by a higher ratio of lipids to proteins than those found in other biological membranes (1). Myelin is distinguished by its high proportion of cholesterol and cerebroside (13). In addition, myelin contains approximately twice as much oleic acid (fatty acid 18:1 cis-9) and more than 5 times the level of long- 
A

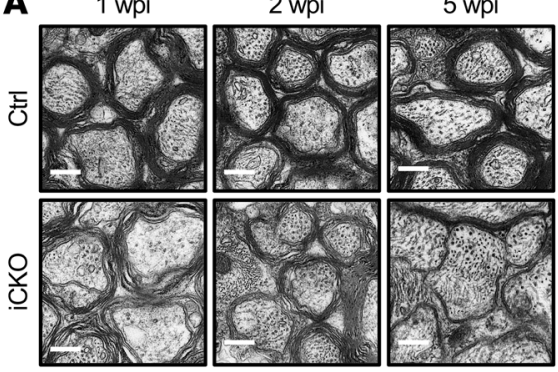

D

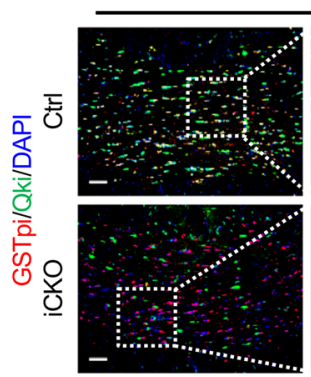

1 wpi

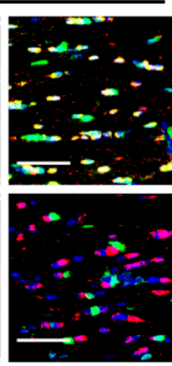

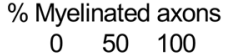

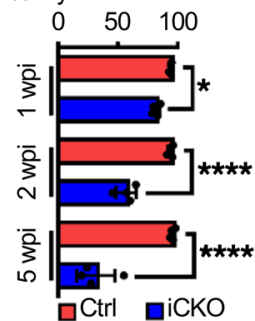

2 wpi
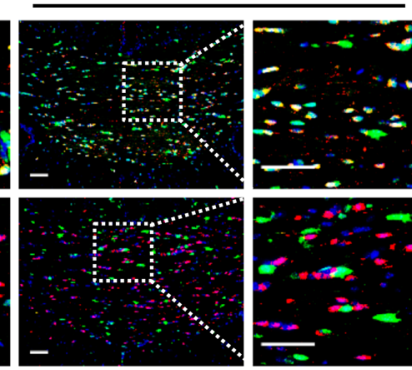

2 wpi

$\mathbf{E}$

1 wpi
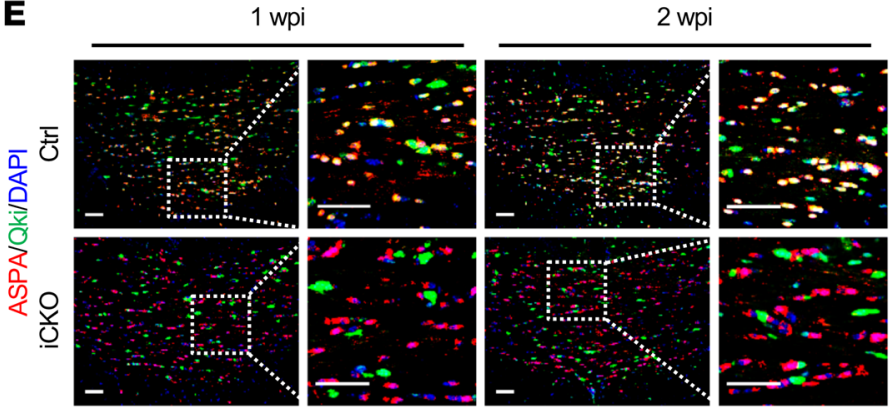

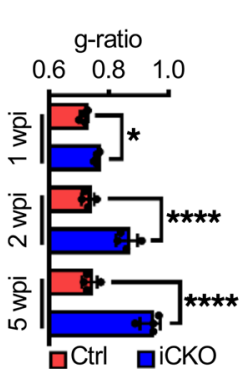

B

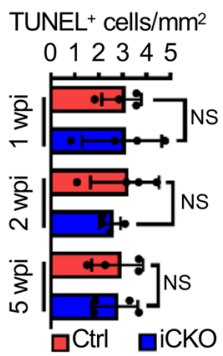

C

Clv-casp-3+ cells $/ \mathrm{mm}^{2}$

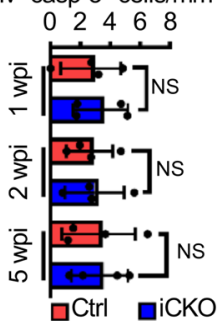

Figure 1. Qki regulates myelin homeostasis independently of oligodendrocyte death. (A) Representative electron micrographs and quantification of the optic nerves of $O k$-iCKO mice and controls (Ctrl) 1, 2, and 5 weeks post tamoxifen injection (wpi). $n=4$ mice/group. Scale bars: $500 \mathrm{~nm}$. (B and C) Quantification of dead cells by TUNEL assay and immunofluorescent staining of cleaved caspase 3 (Clv-casp-3) in the corpus callosum of the experimental mice in $\mathbf{A}$ ( $n=4$ mice/group). ( $\mathbf{D}$ and $\mathbf{E}$ ) Representative images and quantification of immunofluorescent staining of GSTpi-Qki (D) and ASPA-Qki (E) in the corpus callosum of the experimental mice in $\mathbf{A}\left(n=4\right.$ mice/group). Scale bars: $50 \mu \mathrm{m}$. Data are mean $\pm \mathrm{SD}$. ${ }^{*} P<0.05$; ${ }^{* * * *} P<0.0001$ by 2 -way ANOVA with Holm-Sidak multiple-comparisons test. NS, not significant.

chain fatty acids (LCFAs) versus levels found in erythrocyte or hepatocyte membranes (13). It has long been observed that major myelin structural proteins and myelin cholesterol exhibit very slow rates of turnover in rodents, leading to the widely believed presumption for decades that CNS myelin is a static material (14-17). Paradoxically, the discoveries of metabolic enzymes in purified myelin indicate that myelin might be a metabolically dynamic substance with rapid turnover of some of its building blocks $(18,19)$.

The currently available oligodendrocyte-specific genetic demyelinating mouse models are all induced or accompanied by oligodendrocyte apoptosis, rendering these models unsuitable for studying myelin turnover per se $(3,4,20-22)$. Unlike those proteins involved in oligodendrocyte survival (20-22), quaking (Qki, encoded by the gene $Q k$, which undergoes alternative splicing to express Qki-5, Qki-6, and Qki-7), a known regulator of oligodendrocyte differentiation and myelination (23-28), does not regulate cell death (29). In addition, our recent analyses suggested that Qki regulates an array of metabolic pathways in neural stem cells
(NSCs) (29), raising the possibility that Qki might be involved in the metabolic turnover of myelin structural components.

In this study, we used an oligodendrocyte-specific $Q k$ conditional knockout mouse model as a tool to address the following questions: (i) Are the structural components of mature myelin static or dynamic? (ii) Is the continuously active metabolism of myelin components required for the maintenance of myelin integrity in adulthood? (iii) Does interruption of myelin metabolic homeostasis play a pathogenic role in demyelinating diseases? Here, we found that structural lipid components of mature myelin are highly dynamic and their turnover is tightly controlled by Qki via coactivation of the peroxisome proliferator-activated receptor $\beta$ retinoid X receptor $\alpha$ (PPAR $\beta$-RXR $\alpha$ ) complex. A continuous supply of the myelin lipids is essential for the maintenance of myelin integrity in adulthood. Our data reveal a previously underappreciated role of lipid metabolism in demyelinating diseases and pave the way for future development of novel disease-modifying therapies that minimize or even reverse myelin loss by modulating lipid biosynthesis in mature oligodendrocytes. 
A

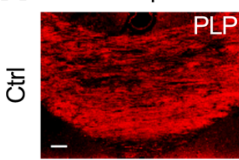

$\frac{\bigcup}{}$

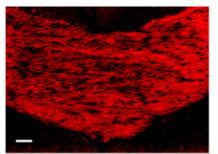

B

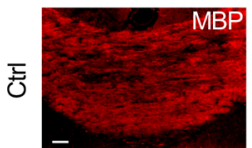

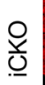

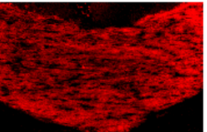

C $\quad 1 \mathrm{wpi}$

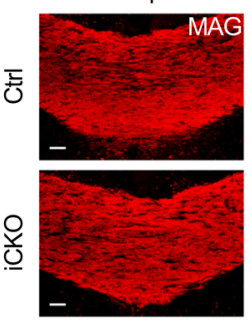

G

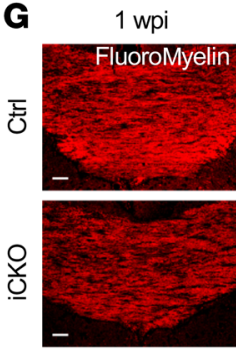

H Spinal cord

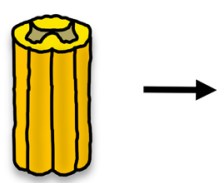

J
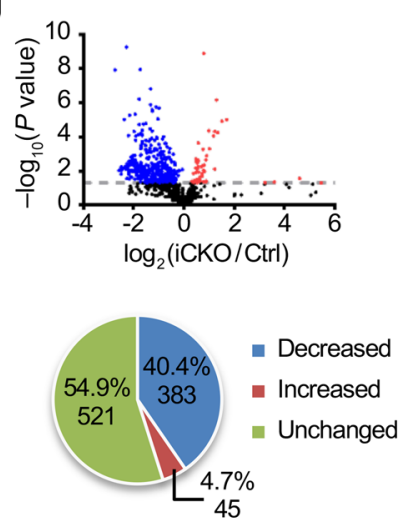

2 wpi
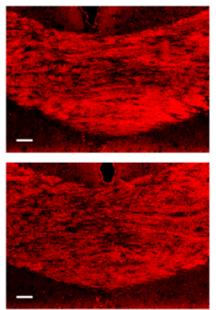

2 wpi

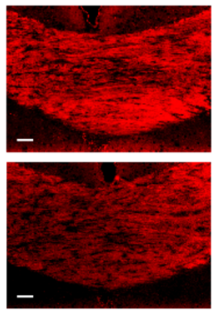

2 wpi

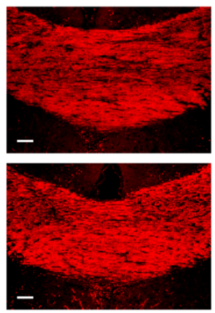

2 wpi

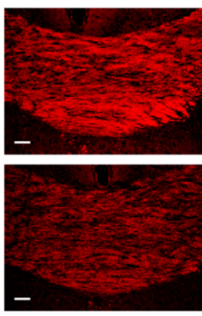

Relative PLP level

0.00 .40 .81 .2

D

Optic nerve
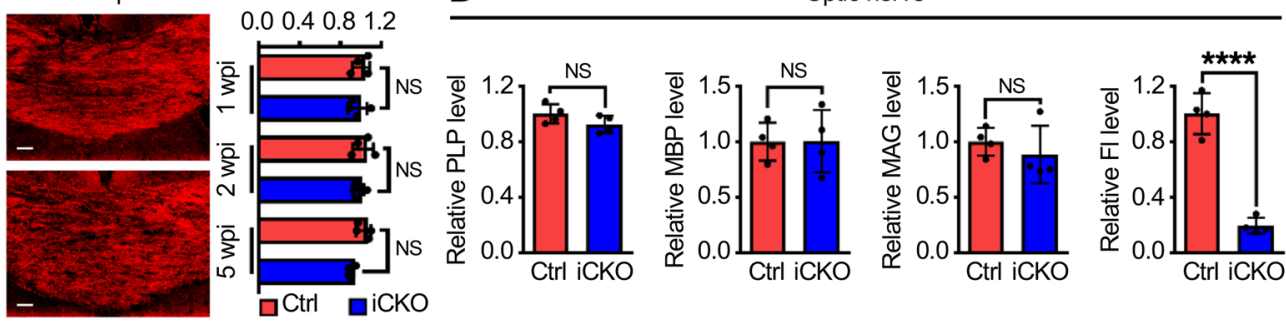

5 wpi

Relative MBP level E

Spinal cord
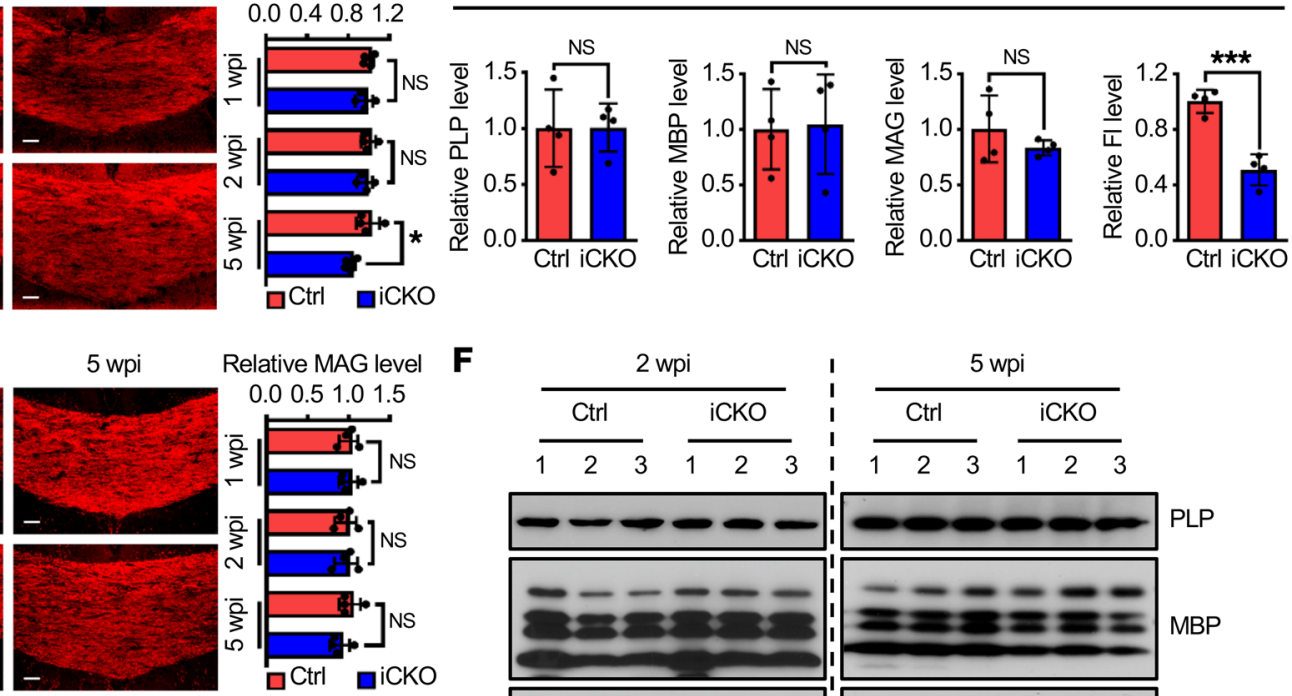

$\mathbf{F}$
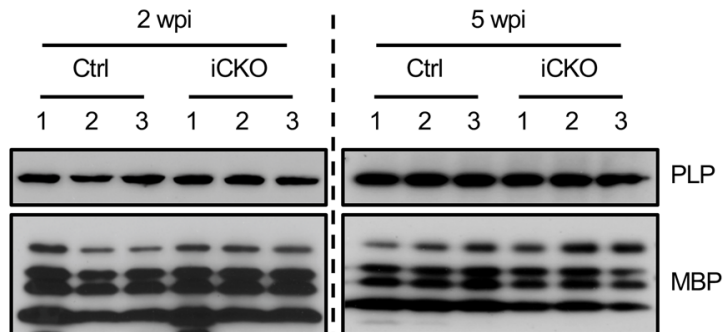

Relative FI level

0.00 .40 .81 .2
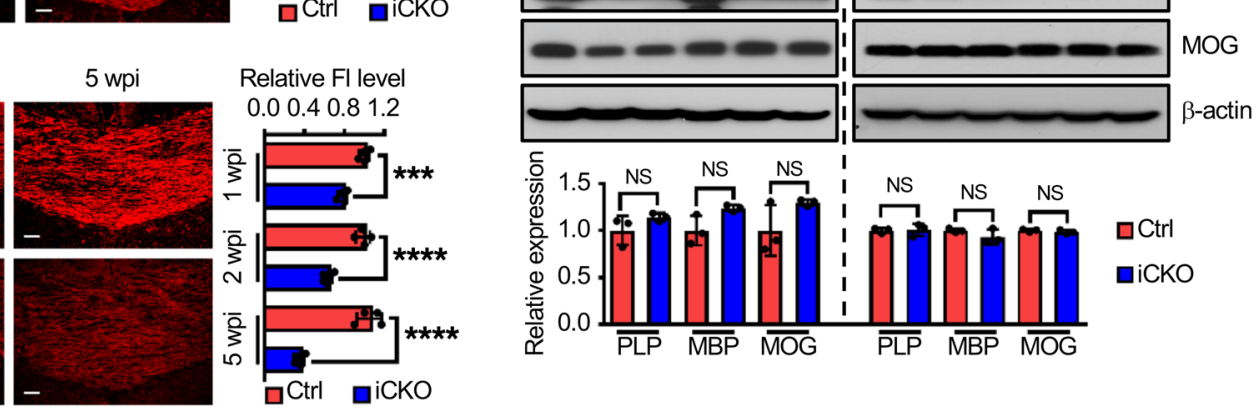

\section{iCKO}

Lipid extraction QTRAP 5500 LC-MS/MS system

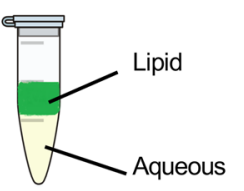

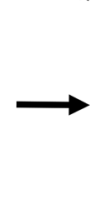

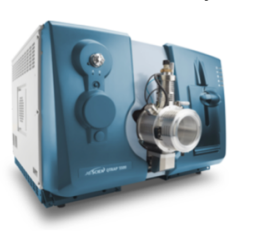

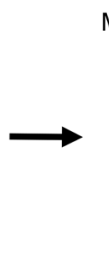
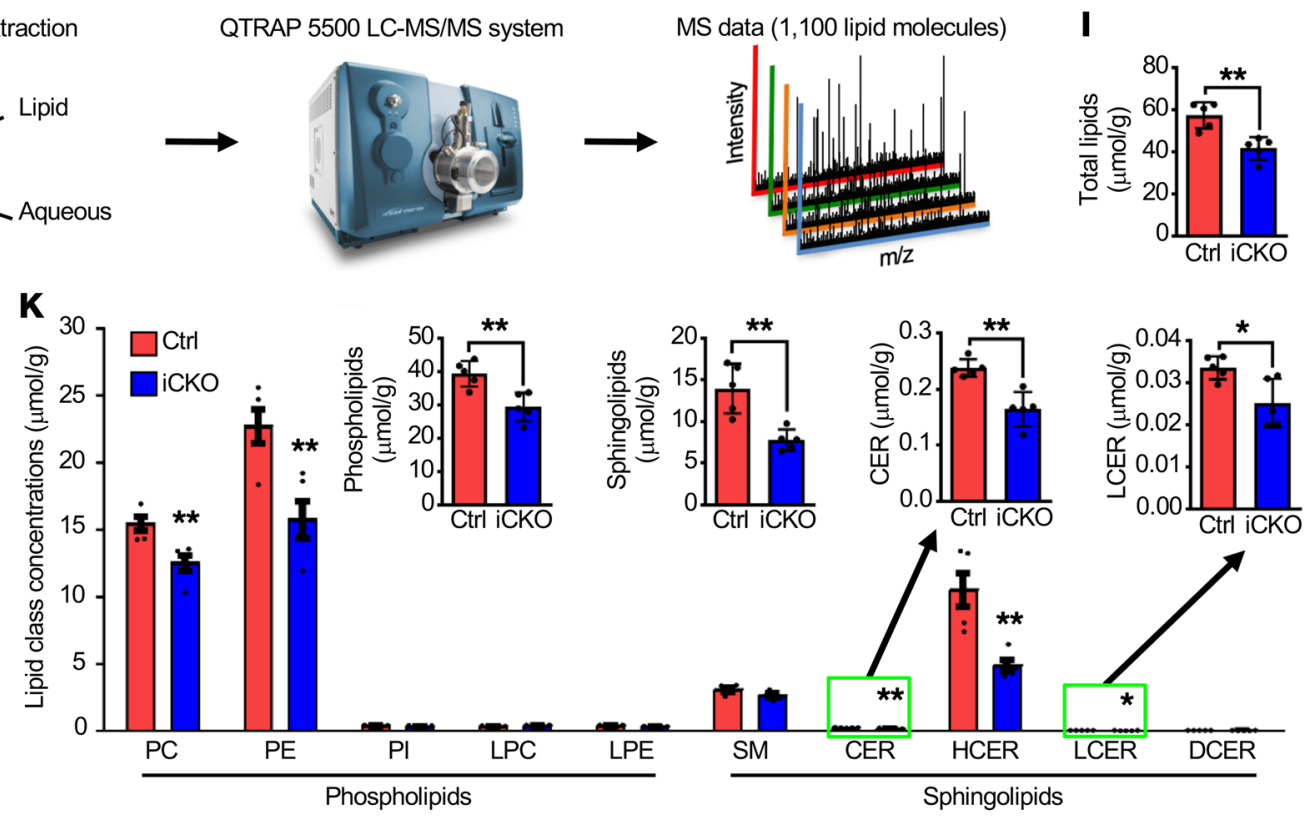
Figure 2. Loss of Qki interrupts mature myelin lipid homeostasis. (A-C) Representative images and quantification of PLP (A), MBP (B), and MAG (C) in the corpus callosum of $Q k$-iCKO mice and controls 1,2 , and 5 weeks post injection (wpi) ( $n=4$ mice/group). Scale bars: $50 \mu \mathrm{m}$. (D and E) Quantification of staining of PLP, MBP, MAG, and FluoroMyelin in the optic nerves (D) and spinal cords (E) of $Q k$-iCKO mice and controls 5 wpi ( $n=4$ mice/group). Fl, FluoroMyelin. (F) Immunoblots and quantification showing the expression of PLP, MBP, and myelin oligodendrocyte glycoprotein (MOG) of $Q k$-iCKO mice and controls 2 and 5 wpi ( $n=3$ mice/ group). (C) Representative images and quantification of staining of FluoroMyelin in the corpus callosum of $Q k$-iCKO mice and controls 1,2 , and $5 \mathrm{wpi}$ ( $n=4$ mice/group). Scale bars: $50 \mu \mathrm{m}$. (H) Schema depicting the workflow for lipidomic analyses. (I) Quantification of the concentration ( $\mu \mathrm{mol} / \mathrm{g}$ tissue) of the total lipids measured by mass spectrometry in the spinal cords of $Q k$-iCKO mice and controls 5 wpi ( $n=5$ mice/group). (J) Volcano plot (top) illustrating the alterations in the concentrations of each lipid molecule in the samples in I ( $n=5$ mice/group). The dotted line represents $P=0.05$. Pie graph (bottom) illustrating the numbers and percentages of significantly decreased, increased, and unchanged molecules in Qk-iCKO mice relative to controls. (K) Quantification of the concentrations of lipid subclasses in the samples in I ( $n=5$ mice/group). PC, phosphatidylcholine; PE, phosphatidylethanolamine; PI, phosphatidylinositol; LPC, lysophosphatidylcholine; LPE, lysophosphatidylethanolamine; SM, sphingomyelin; CER, ceramide; HCER, hexosylceramide; LCER, lactosylceramide; DCER, dihydroceramide. Data are mean \pm SD. ${ }^{*} P<0.05$; ${ }^{* *} P<0.01$; ${ }^{* *} P<0.001$; ${ }^{* * *} P<0.0001$ by 2 -way ANOVA with Holm-Sidak multiple-comparisons test (A-C and $\mathbf{G}$ ) or Student's $t$ test (D-F, I, and $\mathbf{K})$. NS, not significant.

\section{Results}

Qki regulates myelin homeostasis independently of oligodendrocyte death. To determine whether Qki is required for mature oligodendrocyte survival and myelin maintenance, we deleted $Q k$ in mature myelinating oligodendrocytes by injecting $P l p-C r e E R^{\mathrm{T} 2} Q k^{f / f l}$ mice (hereafter called $Q k$-iCKO mice) with tamoxifen at age 8 weeks the time by which the myelin structure is fully established (Supplemental Figure 1, A and B; supplemental material available online with this article; https://doi.org/10.1172/JCI131800DS1) (1). Approximately 2 weeks after tamoxifen injection, $Q k$-iCKO mice progressively developed tremors, splayed gait, ataxia, and hind limb paresis that culminated in paralysis with hunched posture, hyperpnea, significant weight loss, and early lethality (Supplemental Figure 1, C-F, and Supplemental Video 1). In contrast, both Plp-CreER ${ }^{\mathrm{T} 2} \mathrm{Qk}^{f l /+}$ mice and wild-type (WT) mice displayed no neurological signs; therefore, both cohorts were used as controls.

Electron microscopy-based ultrastructural analyses of the optic nerves revealed that demyelination occurred as early as 1 week after $Q k$ deletion. We found that $18.2 \%, 43.1 \%$, and $68.4 \%$ of the axons in $Q k$-iCKO mice were completely devoid of myelin sheaths 1, 2, and 5 weeks after tamoxifen injection, respectively, whereas about $95 \%$ of the axons in controls were wrapped by compact myelin (Figure 1A and Supplemental Figure 1G). Moreover, the myelin sheaths surrounding spared axons in $Q k-i C K O$ mice became gradually thinner than did those in controls (ratio of the inner axonal diameter to the total outer diameter [g-ratio]: 0.76 versus $0.72,0.86$ versus 0.73 , and 0.94 versus 0.74 at 1,2 , and 5 weeks after tamoxifen injection, respectively; Figure 1A). Similarly, the percentages of myelinated axons and the thicknesses of myelin sheaths in the corpus callosum and spinal cords of $Q k$-iCKO mice were also significantly reduced compared with those in the respective CNS regions of control mice (Supplemental Figure 1, H and I), revealing that Qki has a global impact on the mature myelin maintenance across multiple CNS regions. In addition, irregular nodes of Ranvier were frequently observed in the optic nerves of $Q k$-iCKO mice (Supplemental Figure 1, J and $\mathrm{K})$, and the optic nerves of $Q k$-iCKO mice had an approximately $54.5 \%$ decrease in their conduction velocities compared with the control mice (Supplemental Figure 1, L-N). As the morphological characteristics, diameter, and density of axons remained unaffected in the optic nerves of $Q k$-iCKO mice (Supplemental Figure 1, $\mathrm{O}$ and $\mathrm{P}$ ), these data suggest that demyelination itself is the cause of the progressive neurological deficits and increased mortality of Qk-iCKO animals. Besides, we also observed demyelination-induced gliosis and inflammation, including upregulation of $\mathrm{GFAP}^{+}$ activated astrocytes and $\mathrm{IBA}^{+}$microglia and infiltration of $\mathrm{CD}^{+}$ $\mathrm{T}$ cells, in the corpus callosum of $Q k$-iCKO mice (Supplemental Figure 1, Q-S).

We next determined whether demyelination induced by Qki depletion occurs in an oligodendrocyte death-independent manner. Neither a TUNEL assay nor cleaved caspase 3 staining revealed a significant difference in the number of apoptotic cells in Qk-iCKO mice and controls across multiple CNS regions (Figure 1, B and C, and Supplemental Figure 2, A and B), and the numbers of oligodendrocytes in the corpus callosum, optic nerves, and spinal cords of $Q k$-iCKO mice were similar to those in the respective CNS regions of controls (Figure 1, D and E, and Supplemental Figure 2, C-G). More importantly, more than $97 \%$ of the oligodendrocytes still lacked Qki expression 5 weeks after tamoxifen injection (Figure 1, D and E, and Supplemental Figure 2, C-G), indicating that most cells were the resident oligodendrocytes with $Q k$ deletion but not the newly differentiated oligodendrocytes from oligodendrocyte precursor cells (OPCs), which were positive for Qki expression (Supplemental Figure 2H). Therefore, although the number of proliferating OPCs was higher in $Q k$-iCKO mice 5 weeks after tamoxifen injection than that in controls (Supplemental Figure 2I), it appears that these OPCs had not differentiated into mature oligodendrocytes. In summary, the observation of severe demyelination without overt oligodendrocyte death in $Q k$-iCKO animals indicates that mature myelin itself is not static, providing what we believe is a unique mouse model to study oligodendrocyte deathindependent myelin homeostasis.

Qki governs mature myelin lipid homeostasis. To determine how myelin homeostasis is disturbed in $Q k$-iCKO mice, we measured the effect of Qki depletion on major myelin structural proteins, including myelin proteolipid protein (PLP), myelin basic protein (MBP), and myelin-associated glycoprotein (MAG). Unexpectedly, immunofluorescent staining revealed only slightly lower levels of these proteins in the corpus callosum of $Q k$-iCKO mice than in controls (Figure 2, A-C) and unchanged levels in the optic nerves and spinal cords (Figure 2, D and E). Similar results were confirmed by immunoblotting in $Q k$-iCKO mice and controls 2 and 5 weeks after tamoxifen injection (Figure 2F). However, the degenerative myelin, indicated by staining with the QD-9 antibody, was dramatically increased in the corpus callosum (85.7-fold), optic nerves (39.2-fold), and spinal cords (9.3-fold) of $Q k$-iCKO mice relative to those in the respective CNS regions of control mice (Supplemental Figure 3). The QD-9 antibody is specific for 
A 8-week-old mice

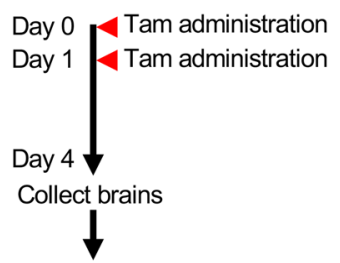

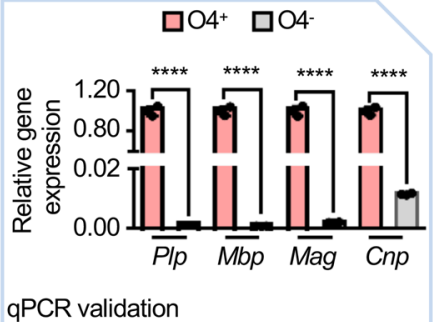

Proteoglycans in cancer
pluripotency of stem cells

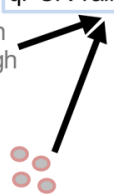

Melanogenesis
Purine metabolism

Hypertrophic cardiomyopathy (HCM)

Hippo signaling pathway

Gap junction

$\begin{array}{llrr}0 & 10 & 20 & 30 \\ & -\log _{10}(P \text { value }) & \end{array}$

B

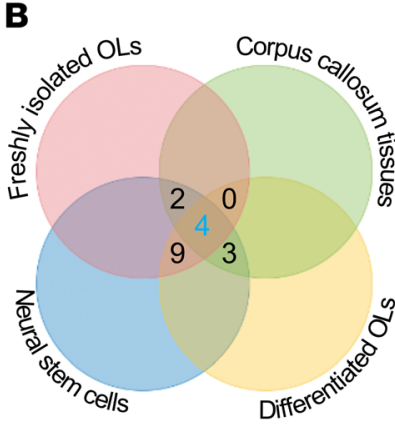

C

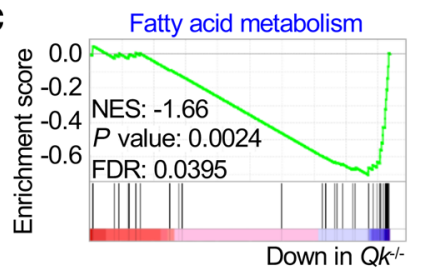

Fatty acid metabolism (15)

Biosynthesis of unsaturated fatty acids (10) Metabolic pathways (30) PPAR signaling pathway (14)
D

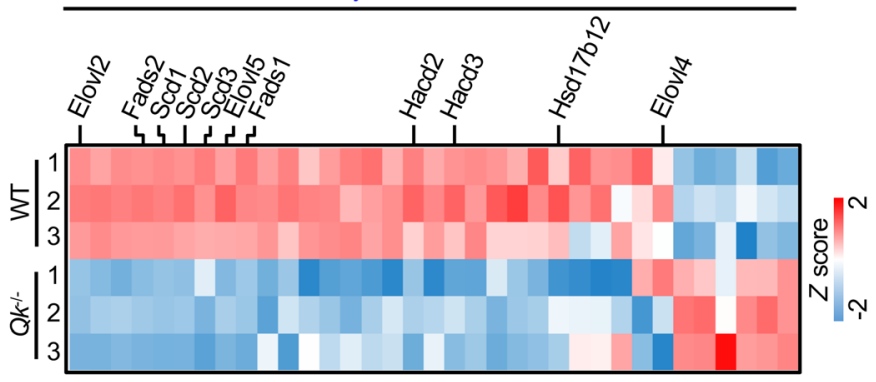

$\mathbf{E}$

Fatty acid desaturation

Fatty acid elongation

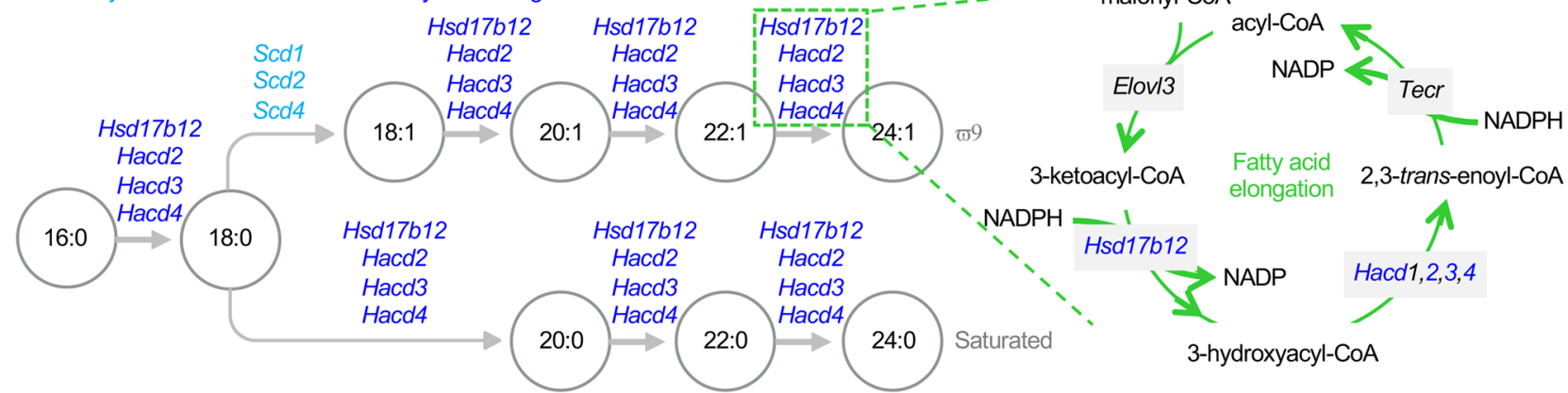

$\mathbf{F}$

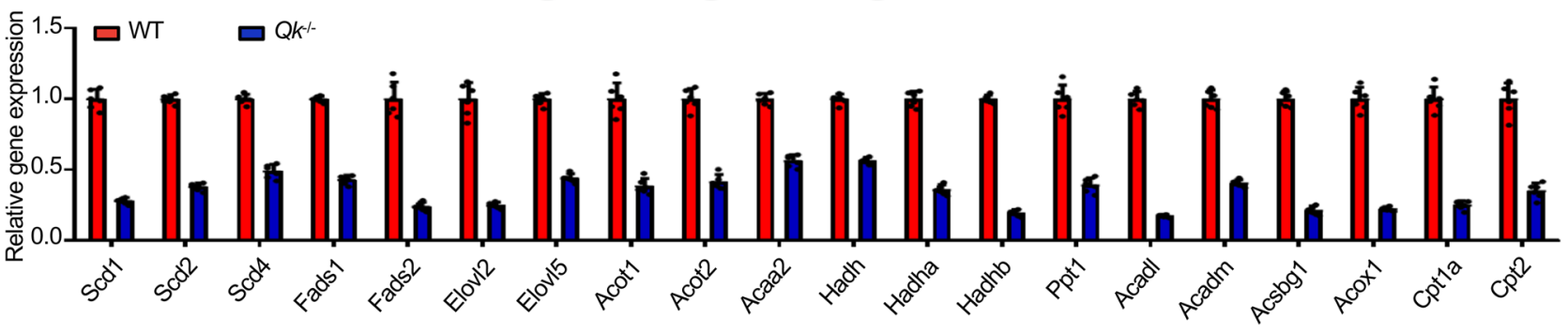

Figure 3. Qki regulates the transcription of genes involved in fatty acid metabolism. (A) Schema depicting the workflow for the fresh isolation of oligodendrocytes from $Q k$-iCKO mice and controls for RNA-seq (left). $n=3$ mice/group. After being validated by RT-qPCR (middle), the $04^{+}$oligodendrocytes (OLs) were used for RNA-seq. Bar graph (right) shows the top 20 enriched pathways. Tam, tamoxifen. (B) Venn diagram depicting the overlap among the top 50 enriched pathways identified from the RNA-seq of freshly isolated mouse OLs, differentiated OLs, NSCs, and adult corpus callosum. The numbers of common genes overlapped in more than 3 cell/tissue types are shown next to individual pathways. (C) GSEA shows the fatty acid metabolism signature in freshly isolated mouse oligodendrocytes. NES, normalized enrichment score; FDR, false discovery rate. (D) Heatmap representing the differentially expressed genes involved in fatty acid metabolism in freshly isolated OLs. (E) Schema depicting desaturating and elongating reactions of fatty acids with the corresponding enzymes. The reduction in fatty acid desaturases is shown in light blue, and the reduction in enzymes of the fatty acid elongation cycle is shown in dark blue. (F) RT-qPCR validation of the differentially expressed genes involved in fatty acid metabolism in WT and Q $\mathrm{ki}^{-}$-depleted ( $\mathrm{k}^{-{ }^{--}}$) differentiated oligodendrocytes. Data are representative of 3 independent experiments $(\mathbf{F})$. Data are mean \pm SD. ${ }^{* * *} P<0.0001$ by Student's $t$ test. 
the exposed epitope of MBP, QDENPVV, which is usually hidden due to the electrostatic interactions of MBP with lipids $(30,31)$. Therefore, we hypothesized that the drastic epitope unmasking of MBP in $Q k-i C K O$ mice might be due to the dissociation of MBP and myelin lipids. Because $70 \%$ of the dry mass of myelin is composed of lipids (1), we next measured total myelin lipid levels using FluoroMyelin, a lipophilic dye with a high selectivity for myelin (32); we observed dramatically lower lipid levels in the corpus callosum (32.7\%), optic nerves (19.5\%), and spinal cords $(50.8 \%)$ of Qk-iCKO mice 5 weeks after tamoxifen injection than in controls (Figure 2, D, E, and G). More importantly, the reduction in myelin lipids in $Q k$-iCKO mice was observed as early as 1 week after tamoxifen injection (Figure $2 \mathrm{G}$ ), which was much earlier than gliosis, $\mathrm{T}$ cell infiltration, and the slight reduction in major myelin structural proteins (Figure 2, A-C, and Supplemental Figure 1, $\mathrm{Q}-\mathrm{S})$, suggesting that autonomous myelin lipid reduction is the primary event after Qki depletion and that Qki may control myelin integrity through regulating lipid metabolism.

To identify the lipid species affected by Qki depletion, we quantified lipid concentrations in the spinal cords of $Q k-i C K O$ mice and controls using mass spectrometry-based lipidomic analyses (Figure 2H and Supplemental Tables 1 and 2). Consistent with the results obtained using FluoroMyelin staining, the overall lipid level in the spinal cords of $Q k$-iCKO mice 5 weeks after tamoxifen treatment was only $72.5 \%$ of that detected in controls (Figure 2I). The concentrations of 428 (45.1\%) of the 949 lipid molecules detected by mass spectrometry were significantly altered after Qki depletion, and the levels of 383 (40.4\%) were significantly reduced after Qki depletion (Figure 2J). Within the entire lipid composition, the total concentration of neutral lipids did not change significantly after Qki depletion $(4.14 \mu \mathrm{mol} / \mathrm{g}$ tissue in controls versus 4.48 $\mu \mathrm{mol} / \mathrm{g}$ tissue in $Q k$-iCKO mice; $P=0.68)$. In contrast, the concentrations of phospholipids and sphingolipids, the 2 major lipid types in myelin, in $Q k$-iCKO mice were $74.6 \%$ and $55.9 \%$, respectively, of the levels in controls (Figure $2 \mathrm{~K}$ ). Taken together, these findings indicate that Qki may control mature myelin maintenance by regulating myelin lipid metabolism.

Qki regulates myelin fatty acid metabolism. To understand how Qki regulates myelin lipid metabolism, we performed transcriptomic analyses by comparing the differentially expressed genes in freshly isolated oligodendrocytes from $Q k$-iCKO mice and controls, in the corpus callosum from $Q k$-iCKO mice and controls, in WT and Qki-depleted oligodendrocytes that differentiated from NSCs, and in WT and Qki-depleted NSCs (Figure 3A and Supplemental Figure 4, A-C). Strikingly, our comparative pathway analyses revealed that the biological processes that were most affected after Qki depletion were involved in fatty acid metabolism, particularly the biosynthesis of unsaturated fatty acids and fatty acid elongation (Figure 3, A-E, and Supplemental Figure $4, \mathrm{~A}-\mathrm{C})$. The transcriptional downregulation of these fatty acid metabolism genes after Qki depletion was verified using realtime quantitative PCR (RT-qPCR) (Figure 3F and Supplemental Figure $4 \mathrm{D}$ ), indicating that the fatty acid metabolism genes are bona fide downstream targets of Qki.

Fatty acid moieties are the major contributors to lipid molecule complexity. Indeed, consistent with the transcriptional regulation of fatty acid metabolism pathways by Qki, further analysis of the lipidomic data revealed that the overall fatty acid level in the spinal cords of $Q k$-iCKO mice was only $72.2 \%$ of that in controls (Figure 4A). Fatty acids can be classified by saturation status and by carbon chain length. We identified the fatty acids whose concentrations were the most substantially reduced by Qki depletion using each of these 2 classifications.

First, the fatty acids were sorted based on their saturation status into 3 groups: saturated fatty acids (SFAs), monounsaturated fatty acids (MUFAs), and polyunsaturated fatty acids (PUFAs); MUFA levels decreased the most after Qki depletion (to 54.2\% of levels in controls; Figure 4B and Supplemental Figure 5, A-D). Because fatty acid composition is crucial for the fluidity and rigidity of the membrane structure of myelin (33), we then determined whether the fatty acid composition was altered in the spinal cords of $Q k$-iCKO mice (Figure $4 \mathrm{C}$ and Supplemental Figure $5 \mathrm{E}$ ). The composition analysis revealed that the percentage of MUFAs among all fatty acids in $Q k$-iCKO mice was lower than that in controls (41.0\% in controls versus $30.8 \%$ in $Q k$-iCKO mice; $P=1.16 \times$ $\left.10^{-5}\right)$, and the percentage of SFAs among all fatty acids in $Q k$-iCKO mice was higher than that in controls $(43.8 \%$ in controls versus $49.5 \%$ in $Q k$-iCKO mice; $\left.P=1.21 \times 10^{-5}\right)$. Accordingly, the ratio of MUFAs to SFAs in $Q k$-iCKO mice was significantly lower than that in controls ( 0.94 in controls versus 0.62 in $Q k$-iCKO mice; $P=1.26$ $\left.\times 10^{-5}\right)$, indicating that the desaturating reactions in converting SFAs to MUFAs are impaired in $Q k$-iCKO mice (Figure 4, D and E). For example, the ratio of oleic acid (18:1) to stearic acid (18:0) in $Q k$-iCKO mice was significantly lower than that in controls $(1.84$ in controls versus 1.32 in $Q k$-iCKO mice; $P=0.00003$; Figure $4 \mathrm{~F}$ ), indicating that this critical desaturating reaction is compromised in $Q k$-iCKO mice. This finding is consistent with the aforementioned transcriptional downregulation of stearoyl-CoA desaturases 1, 2, 3, and $4(S c d 1,-2,-3$, and -4$)$, genes that encode the enzymes that catalyze the desaturation of stearic acid, after Qki depletion (Figure 3D and Figure 4E).

Second, an analysis of the fatty acids based on their carbon chain lengths revealed that the levels of LCFAs and very-longchain fatty acids (VLCFAs), but not medium-chain fatty acids (MCFAs), were significantly diminished after Qki depletion (Supplemental Figure 5, F-H). The composition analysis further indicated that the percentage of VLCFAs among the total fatty acids was the most diminished after Qki depletion (8.6\% in controls versus $5.3 \%$ in Qk-iCKO mice; $P=0.0049$ ). Accordingly, the fatty acid ratios of 20:0/18:0, 20:1/18:1, 24:1/22:1, 22:5/20:5, and 22:4/20:4 were all significantly reduced after Qki depletion (Figure 4, E and F, and Supplemental Figure 5, I and J), suggesting that these elongating reactions are impaired. These results are consistent with the aforementioned transcriptional downregulation of hydroxysteroid 17- $\beta$ dehydrogenase 12 ( $H s d 17 b 12)$ and 3-hydroxyacyl-CoA dehydratase 2, 3, and 4 (Hacd2, -3 , and -4), genes that encode the enzymes responsible for the elongation of these fatty acids, after Qki depletion (Figure 3D, Figure 4E, and Supplemental Figure 5I).

In summary, complex lipidomic analyses revealed that the most markedly reduced fatty acids after Qki depletion were the SFAs, MUFAs, LCFAs, and VLCFAs (Supplemental Figure 5, B, $\mathrm{C}, \mathrm{G}$, and $\mathrm{H}$ ). Our finding that Qki is a transcriptional regulator of fatty acid metabolism raised the strong possibility that impaired lipid metabolism is a cause of the Qki depletion-induced demye- 

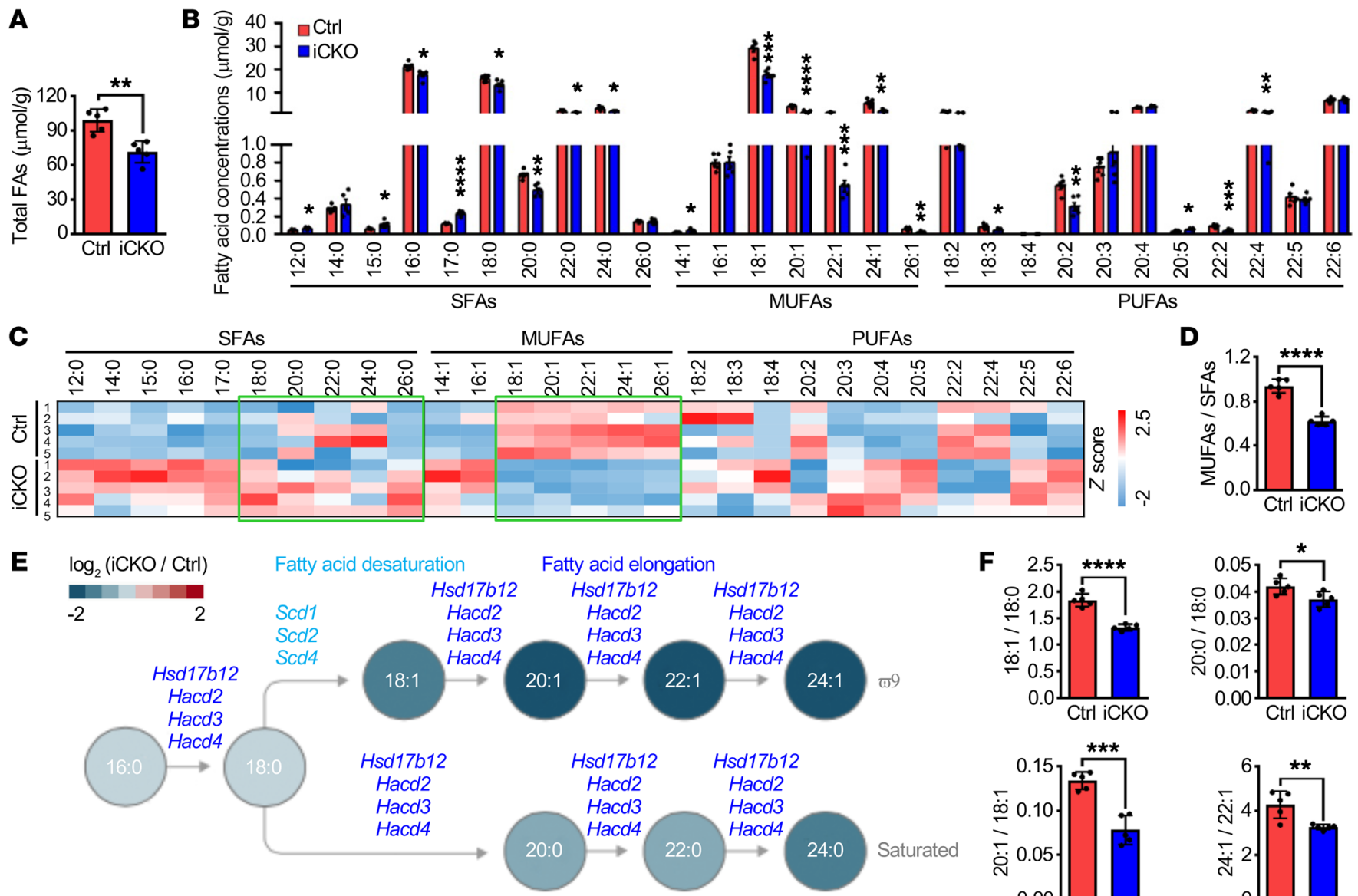

Fatty acid elongation
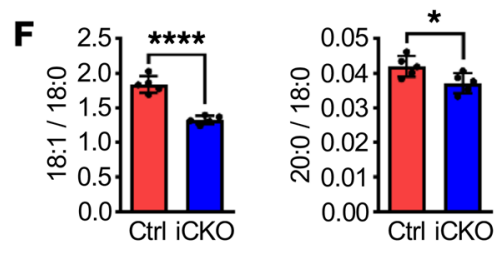

\section{G}

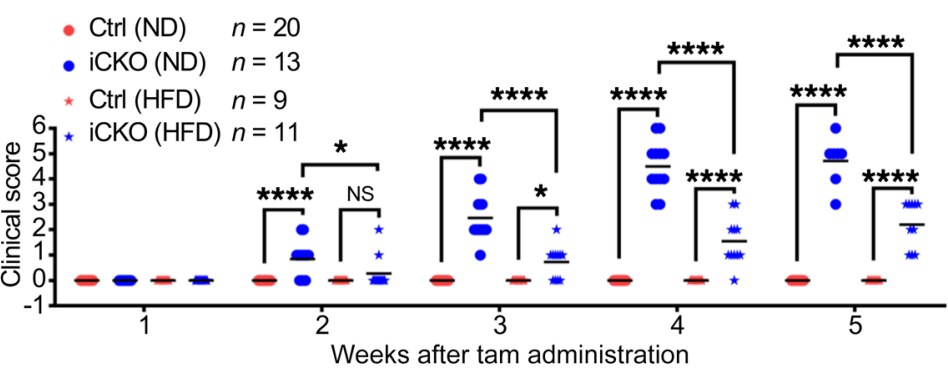

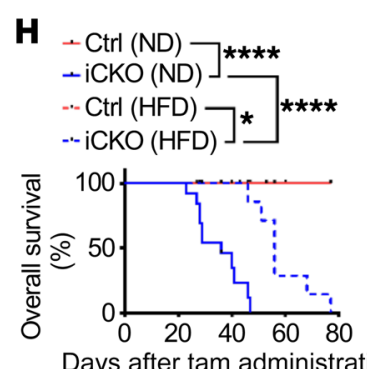

Days after tam administration

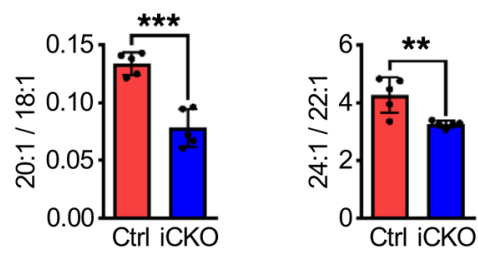

J

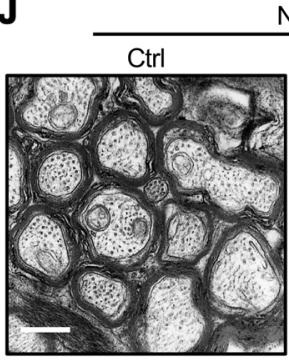

ND
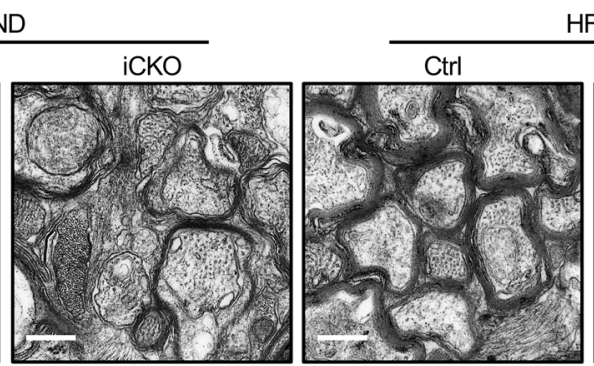

HFD

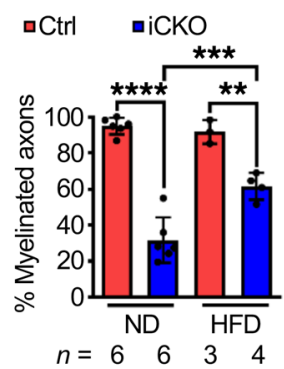

$\mathbf{K}$

ND

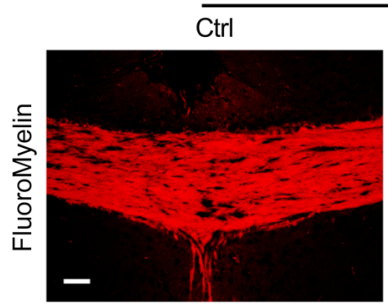

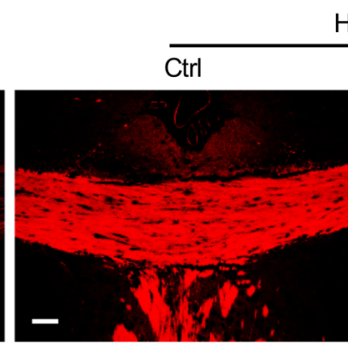

HFD

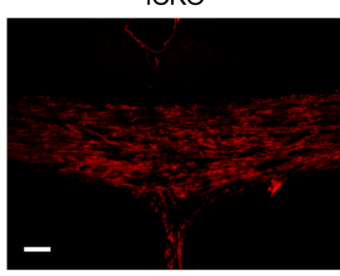

$\square$ Ctrl $=$ iCKO

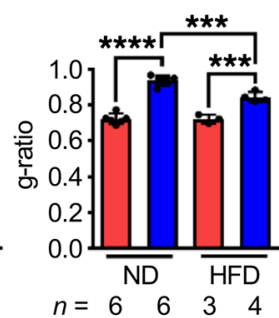

I aCtrl iCKO

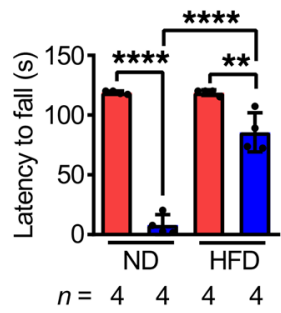

aCtrl -iCKO

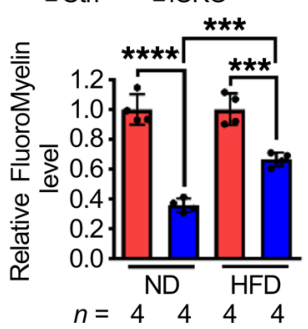


Figure 4. Qki controls fatty acid desaturation and elongation and HFD alleviates Qki deficiency-induced demyelination. ( $A$ and $\mathbf{B}$ ) Quantification of the concentration of total and each species of fatty acids (FAs) in the spinal cords of $Q k$-iCKO mice and controls 5 weeks post injection (wpi) ( $n=5$ mice/group). (C) Heatmap plotting the compositions of each fatty acid species in the samples in $\mathbf{A}$ ( $n=5$ mice/group). The green boxes highlight the saturated and monounsaturated LCFAs and VLCFAs. (D) Quantification of the MUFA/SFA ratios in the samples in $\mathbf{A}(n=5$ mice) group). (E) Schema showing the desaturating and elongating reactions of fatty acids with the reduction in specific fatty acid molecules (from lipidomic data) and the downregulated corresponding enzymes (from RNA-seq data) in Ok-iCKO mice relative to controls. (F) Quantification of the product/substrate ratios of representative fatty acid desaturating and elongating reactions in the samples in $\mathbf{A}$ ( $n=5$ mice/group). ( $(\mathbf{C}$ and $\mathbf{H}$ ) The clinical scores (C) and Kaplan-Meier overall survival curves (log-rank test; $\mathbf{H}$ ) of $Q k$-iCKO mice and controls fed a normal diet (ND) or HFD. The experimental mouse number is indicated in G. (I) Latency to fall (in seconds) off the rotarod ( $5 \mathrm{rpm}$ ) for $\mathrm{O} k$-iCKO mice and controls fed an ND or HFD for 5 wpi. (J) Representative electron micrographs and quantification of the percentage of myelinated axons and g-ratio of the optic nerves of $Q k$-iCKO mice and controls fed an ND or HFD for 5 wpi. Scale bars: 500 $\mathrm{nm}$. (K) Representative images and quantification of FluoroMyelin level in the corpus callosum of $O k$-iCKO mice and controls fed an ND or HFD for 5 wpi. Scale bars: $50 \mu \mathrm{m}$. Data are mean $\pm \mathrm{SD}$. ${ }^{*} P<0.05$; ${ }^{* *} P<0.01$; ${ }^{* * *} P<$ $0.001 ;{ }^{* * * *} P<0.0001$ by Student's $t$ test $(\mathbf{A}, \mathbf{B}, \mathbf{D}$, and $\mathbf{F})$ or 1-way ANOVA followed by Bonferroni's post hoc test ( $\mathbf{G}$ and $\mathbf{I}-\mathbf{K}$ ). NS, not significant.

linating phenotype. To test this hypothesis, we determined whether dietary supplementation of fatty acids alleviated the severe demyelinating symptoms of $Q k$-iCKO animals. Indeed, compared with the $Q k$-iCKO mice that were fed a normal diet, the mice that received a high-fat diet (HFD) enriched with various fatty acids had a markedly longer time to the onset of neurological signs, lower mean clinical scores, better coordinate movement on rotarod tests, and longer median overall survival durations ( 56 days versus 36 days; Figure 4, G-I, Supplemental Figure 5K, and Supplemental Video 2). Ultrastructural analyses confirmed that $Q k$-iCKO mice fed the HFD had a significantly higher percentage of myelinated axons $(31.7 \%$ in the normal diet group versus $61.5 \%$ in the HFD group; $P<0.0001$ ) and thicker myelin sheaths (g-ratio: 0.94 in the normal diet group versus 0.84 in the HFD group; $P=0.0001$ ) than did mice fed a normal diet (Figure 4J). Moreover, $Q k$-iCKO mice fed the HFD had $85.8 \%$ higher myelin lipid levels than did mice fed a normal diet (Figure 4K and Supplemental Figure 5L). Together, these results demonstrate that a Qki-mediated continuous fatty acid supply is required for mature myelin maintenance and that an HFD can partially rescue Qki loss-induced demyelination.

Qki regulates fatty acid metabolism via PPAR $\beta-R X R \alpha$. The PPAR signaling pathway is a major regulator of lipid metabolism, adipogenesis, and energy homeostasis (34). Interestingly, pathway analyses with transcriptomic data revealed that the PPAR signaling pathway was one of the cascades that was most profoundly affected by Qki depletion (Figure 3B and Figure 5A), suggesting that Qki may regulate lipid metabolism through this pathway. Based on the observations that the expression of all homologs of PPAR in the corpus callosum was comparable in $Q k$-iCKO mice and controls and that most Qki-5 was localized on chromatin (Supplemental Figure 6, A and B), we hypothesized that Qki-5 interacts with the PPAR-RXR complex to modulate the transcription of fatty acid metabolism genes. Indeed, coimmunoprecipitation (co-IP) exper- iments revealed that PPAR $\beta$ and RXR $\alpha$, but not other PPAR and RXR homologs, specifically interacted with Qki-5 (Figure 5, B-D).

Although Qki-5 is an RNA-binding protein (23), the interaction between Qki-5 and PPAR $\beta$ was not affected by either RNase A treatment or mutation of Qki-5 (V157E), which disrupts the RNA-binding ability (27), indicating that RNA was not essential for the formation of the Qki-5-PPAR $\beta$-RXR $\alpha$ complex (Figure 5, E and F, and Supplemental Figure 6C). In fact, approximately $96.5 \%$ of the differentially expressed genes after Qki depletion do not contain a Qki response element (QRE) (Supplemental Figure 6D). In support of this, the transcription of fatty acid metabolism genes was similarly upregulated by ectopically expressed Qki-5 ${ }^{\mathrm{WT}}$ and Qki-5 ${ }^{\mathrm{V} 157 \mathrm{E}}$ (Supplemental Figure 6E), suggesting that Qki regulates gene expression independently of its RNA-binding ability.

To uncover the molecular mechanism by which the QkiPPAR $\beta$-RXR $\alpha$ complex regulates the transcription of genes involved in lipid metabolism, differentiated oligodendrocytes with ectopic expression of hemagglutinin-tagged PPAR $\beta$ (HA-PPAR $\beta$ ) were analyzed by chromatin immunoprecipitation and sequencing (ChIP-seq) using anti-Qki-5, anti-PPAR $\beta$, and anti-HA antibodies, and 30,179 Qki-5-binding peaks, 26,406 PPAR $\beta$-binding peaks, and 30,042 HA-binding peaks were identified (Figure 5G). Consensus PPAR response element (PPRE) motifs were significantly enriched in both PPAR $\beta$ - and HA-binding events (Supplemental Figure 6F), confirming the specificity of the ChIP. Strikingly, we found that $74.4 \%(19,654$ of 26,406$)$ of PPAR $\beta$ sites and $66.9 \%(20,113$ of 30,042$)$ of HA sites were cobound by Qki-5 (Figure 5G). Moreover, genomic distribution analyses of Qki5- and PPARß-binding events showed similarly high enrichment at promoter/transcription start site regions $(30.2 \%, 31.1 \%$, and $28.6 \%$ of Qki-5-, PPAR $\beta$-, and HA-binding events, respectively, whereas only $1.1 \%$ of regions are considered promoter regions across the whole genome; Figure 5, H-J). Reinforcing the notion that Qki-5 and PPAR $\beta$ coregulate the transcription of fatty acid metabolism genes, Qki-5, PPAR $\beta$, HA, and Pol II occupancies were codetected at the promoter regions of 45 genes involved in fatty acid metabolism, such as $S c d 2, S c d 4$, fatty acid desaturase 1 (Fads1), Fads2, ELOVL fatty acid elongase 5 (Elovl5), and Hacd2 (Figure 5K and Supplemental Figure 6G), and the mRNA levels of majority of these genes $(73.3 \%$; 33 of 45$)$ were significantly downregulated in $\mathrm{Qk}^{-/-}$oligodendrocytes relative to WT oligodendrocytes (Figure 3, D-F). Consistently, Qki-5- and PPAR $\beta$-binding events showed similarly high enrichment at promoter/transcription start site regions in freshly isolated mouse oligodendrocytes (Supplemental Figure 6, H-L). Importantly, we found that the promoter occupancies of PPAR $\beta$ at $S c d 2$ and carnitine palmitoyltransferase 1c (Cpt1c) were greatly diminished by Qki depletion (Supplemental Figure 6M), suggesting that Qki either recruits or stabilizes PPAR $\beta$ at the promoters. In addition, we found that the PPAR $\beta$-specific agonist GW501516 stimulated the transcription of fatty acid metabolism genes with comparable potency in WT and $\mathrm{Qk}^{-/-}$oligodendrocytes (Supplemental Figure $6 \mathrm{~N}$ ), indicating that Qki-5 might serve as a transcriptional coactivator instead of an essential component of the PPAR $\beta$-RXR $\alpha$ complex. In support of this, ectopically expressed Qki-5 promoted the transcription of a PPAR-responsive luciferase reporter (Supplemental Figure 6O). Together, these findings strongly suggest that Qki-5 functions as 
A

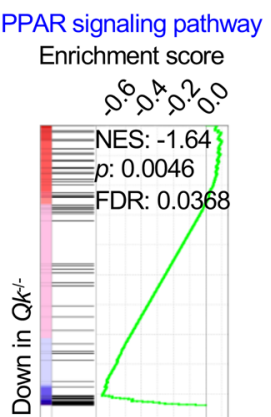

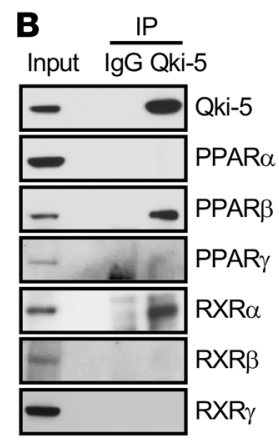

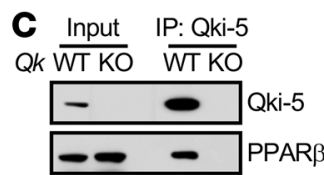

D

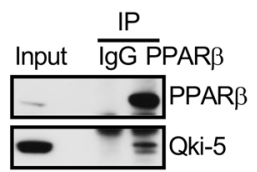

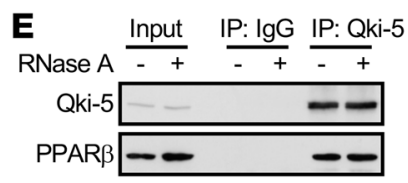

$\mathbf{F}$

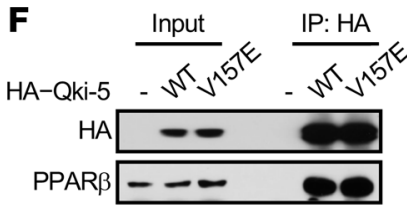

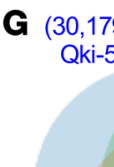

Qki-5

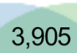

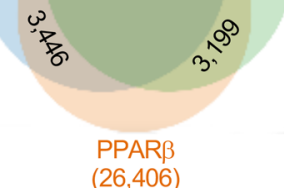

H
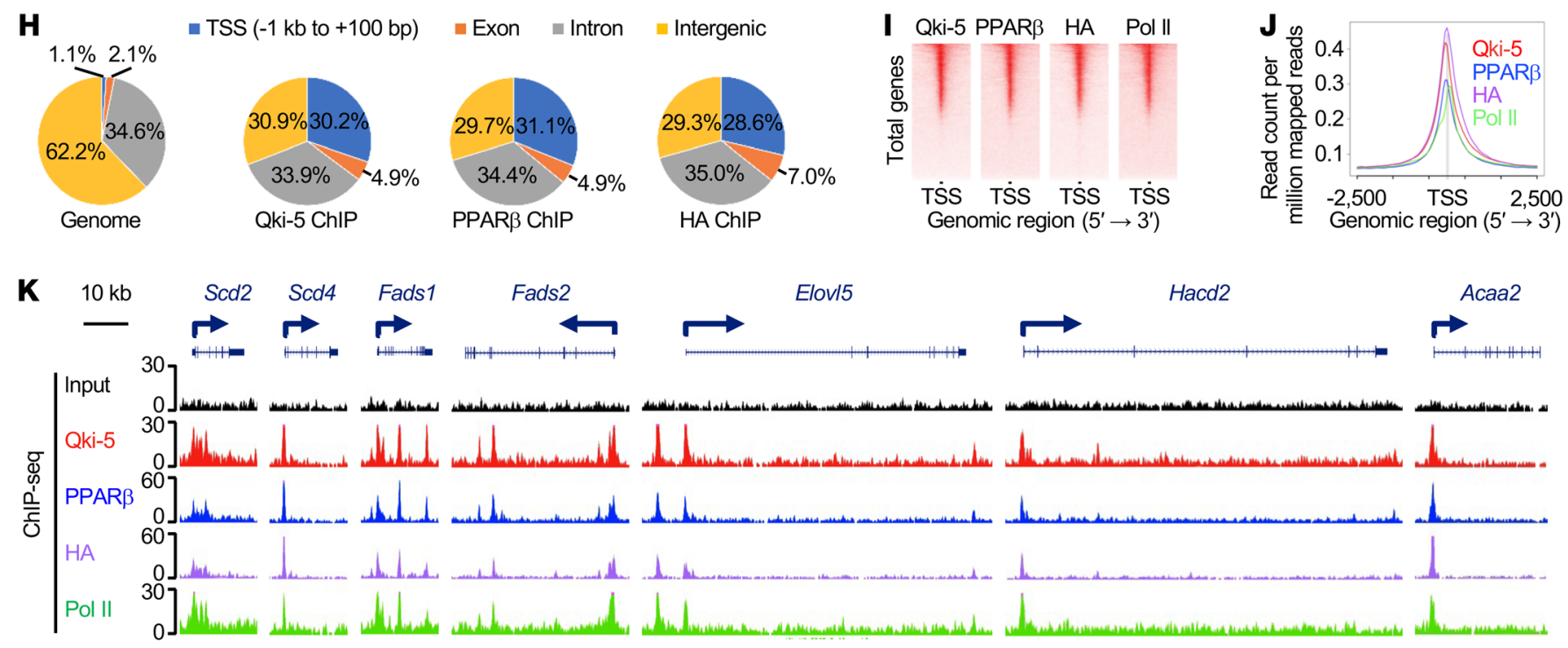

Figure 5. Qki-5 interacts with PPARß-RXR $\alpha$ to regulate the transcription of genes involved in fatty acid metabolism. (A) GSEA shows the PPAR signaling pathway signature in freshly isolated mouse oligodendrocytes. (B) Co-IP using an anti-Qki-5 antibody in differentiated oligodendrocytes, followed by the detection of homologs of PPAR and RXR via immunoblotting. (C) Co-IP using an anti-Qki-5 antibody in differentiated oligodendrocytes, followed by detection of PPAR $\beta$ via immunoblotting. $Q k$-knockout $(\underline{Q} k-K O)$ cells served as a negative control to exclude nonspecific immunoprecipitation from the anti-Qki5 antibody. (D) Reciprocal co-IP using an anti-PPAR $\beta$ antibody, blotted with an anti-Qki-5 antibody. An IgG antibody served as a negative control. (E) Co-IP of Qki-5 and PPAR $\beta$ in differentiated oligodendrocytes that were treated with RNase A while alive. (F) Co-IP of HA-Qki-5 (WT or V157E mutant) and PPAR $\beta$ in differentiated oligodendrocytes. (C) Overlap of ChIP-seq peak sets of Qki-5, PPAR $\beta$, and HA in differentiated oligodendrocytes with ectopic expression of HA-PPAR $\beta$. (H) Genomic global distribution of the ChIP-seq peaks of Qki-5, PPAR $\beta$, and HA. (I) ChIP-seq density heatmaps of Qki-5, PPAR $\beta$, HA, and Pol II within $\pm 1 \mathrm{~kb}$ of the transcription start site (TSS). All peaks are rank ordered from high to low Qki-5 occupancy. (J) Average genome-wide occupancies of Qki-5, PPAR $\beta, H A$, and Pol II within \pm 2.5 kb of the TSS. (K) Representative ChIP-seq binding peaks of Qki-5, PPAR $\beta$, HA, and Pol II on gene loci associated with fatty acid metabolism. Data are representative of 3 independent experiments (B-F).

a coactivator of PPAR $\beta-R X R \alpha$ to regulate the expression of fatty acid metabolism genes that are essential for maintaining mature myelin homeostasis.

$P P A R \beta$ and RXR agonists alleviate Qki deficiency-induced demyelination. A previous study showed that PPAR $\beta$-null mice had altered myelin in the corpus callosum, yet it was unclear whether the effect was cell autonomous and whether PPAR $\beta$ regulates myelin lipid homeostasis (35). To address these questions, we deleted Ppard (gene that encodes PPAR $\beta$ ) in mature myelinating oligodendrocytes by injecting Plp-CreER ${ }^{\mathrm{T} 2} \mathrm{Ppard}^{\mathrm{t} / f l}$ mice (hereafter called Ppard-iCKO mice) with tamoxifen at age 8 weeks (Supplemental Figure 7, A and B). Compared with control mice, at 5 weeks after tamoxifen injection Ppard-iCKO mice displayed significantly reduced motor coordinative movement (Supplemental Figure 7C), completely devoid of myelin sheaths in $36.7 \%$ of the axons, and significant reduction in the thickness of myelin sheaths surrounding the remaining axons (g-ratio: 0.83 versus $0.72 ; P=0.0066$; Supplemental Figure 7, D-G), yet a similar number of oligodendrocytes in the corpus callosum (Supplemental Figure 7, H and I). These results indicate that, similarly to Qki, PPAR $\beta$ is critical for the maintenance of oligodendrocyte death-independent myelin homeostasis. Consistently, depletion of PPAR $\beta$ in mature oligodendrocytes resulted in a significant reduction in myelin lipids but not major myelin structural proteins in the corpus callosum (Supplemental Figure 7, J and K). Together with the data indicating that Qki interacts with PPAR $\beta$ to transcriptionally control myelin lipid metabolism (Figure 5 and Supplemental Figure 6), the similar demyelinating phenotypes of Ppard-iCKO mice and $Q k$-iCKO mice support the model that Qki and PPAR $\beta$ work together in regulating myelin lipid metabolism and controlling myelin homeostasis.

Because loss of Qki diminished but not totally abolished PPAR $\beta-R X R \alpha$ transcriptional activity (Supplemental Figure $6 \mathrm{~N}$ ), 
we next determined whether the CNS-permeant PPAR $\beta$ agonist KD3010 (36) and RXR agonist bexarotene (37) could alleviate Qki depletion-induced demyelinating symptoms. Oral administration of KD3010 or bexarotene in Qk-iCKO mice significantly delayed the onset of neurological deficits, lowered clinical scores, and prolonged median survival times (2.34- or 1.41-fold, respectively) compared with treatment with vehicle (Figure 6, A-D, Supplemental Figure 8, A and B, and Supplemental Videos 3 and 4). Strikingly, 23.1\% (3 of 13) of Qk-iCKO mice treated with KD3010 or bexarotene lived for more than 100 days, with the maximum survival duration increased by 3.72- or 4.03-fold compared with that of $Q k-$ iCKO mice treated with vehicle, respectively (Figure 6, B and D). Consistent with their clinical improvement, $Q k$-iCKO mice treated with KD3010 or bexarotene had $115.3 \%$ or $73.7 \%$ more myelinated axons, with thicker myelin sheaths, than did $Q k$-iCKO mice treated with vehicle (Figure 6, E and F). In support of this, $Q k$-iCKO mice treated with KD3010 or bexarotene had $119.4 \%$ or $89.5 \%$ higher myelin lipid levels than did $Q k$-iCKO mice treated with vehicle (Figure 6, G and H, and Supplemental Figure 8, C and D). Additionally, the reduction in expression of $S c d 1, S c d 2, S c d 4$, and Elovl5 in $Q k-$ iCKO mice was significantly alleviated by treatment with KD3010 (Supplemental Figure 8E). These significant alleviations of Qki depletion-induced demyelinating symptoms and enhancement of myelin lipid biosynthesis by PPAR $\beta$ and RXR agonists confirm that PPAR $\beta$-RXR $\alpha$-dependent fatty acid metabolism is a key downstream target of Qki in maintaining mature myelin homeostasis and provide justification for the use of PPAR $\beta / R X R \alpha$ agonists in future clinical trials for patients with demyelinating diseases.

Myelin lipids are preferentially reduced in MS. Experimental autoimmune encephalomyelitis manifests as an acute ascending paralysis that either remits or plateaus, and is widely used as an animal model of relapsing-remitting MS (38). In contrast, the clinical course of our $Q k$-iCKO mice is characterized by a chronic accumulation of neurological disability that is more reminiscent of the primary progressive subset of MS. To investigate the potential role of myelin lipid dysregulation in the pathogenesis of human demyelinating diseases, we obtained 30 individual lesions that were characterized following the Lassmann/Brück method (39) from the brains of 6 patients with primary progressive MS (PPMS) (Supplemental Figure 9A). Frontal lobe white matter tissues from 5 donors without neurological disease were analyzed in parallel as controls. The average level of myelin lipids in these MS lesions, as measured by FluoroMyelin staining, was $24.8 \%$ of that in the control tissues (Figure 7A). In contrast, despite marked variations among individual lesions, the average levels of the major myelin structural proteins (PLP, MBP, and MAG) were not significantly different in the MS lesions compared with the controls (Figure 7B and Supplemental Figure 9, B and C). By grouping these MS lesions on the basis of their myelin lipid and protein levels, we found that $50 \%$ ( 15 of 30 ) of the lesions exhibited low lipid levels but normal protein levels (Fl' $\left.{ }^{\mathrm{l}} \mathrm{PLP} \mathrm{P}^{\mathrm{hi}}\right), 10 \%$ exhibited normal lipid levels but low protein levels $\left(\mathrm{Fl}^{\mathrm{hi}} \mathrm{PLP} \mathrm{P}^{\mathrm{lo}}\right)$, and the remaining $40 \%$ exhibited low levels of both lipids and proteins $\left(\mathrm{Fl}^{\mathrm{lo}} \mathrm{PLP}^{\mathrm{lo}}\right.$ ) (Figure 7, C-F). These data suggest that myelin lipids are preferentially reduced in a subset of PPMS lesions.

Oligodendrocyte death is often considered a primary pathogenic event in MS (2-4). To determine whether the observed lower levels of myelin lipids in MS lesions were correlated with a lower density of oligodendrocytes, we counted the number of OLIG2+ oligodendrocytes in each MS lesion (Supplemental Figure 9D). We found that $\mathrm{Fl}^{\mathrm{lo}} \mathrm{PLP} \mathrm{P}^{\mathrm{lo}}$ lesions had significantly lower numbers of oligodendrocytes than did control tissues $(P<0.01)$, suggesting that oligodendrocyte death was the major cause of the demyelination and decreased levels of myelin lipids and proteins that were observed in this subgroup of lesions (Figure 7, D and G). In contrast, $80 \%$ (12 of 15 ) of the $\mathrm{Fl}^{\mathrm{lo}} \mathrm{PLP}^{\mathrm{hi}}$ lesions (indicated by the red-dashed box in Figure 7G) had normal oligodendrocyte densities, suggesting that diminished myelin lipid levels, rather than oligodendrocyte death, might be responsible for demyelination in this subgroup of lesions (Figure 7, D and G). The remaining 20\% (3 of 15) of the $\mathrm{Fl}^{\mathrm{lo}} \mathrm{PLP}^{\mathrm{hi}}$ lesions had relatively low oligodendrocyte density but normal myelin protein staining. One explanation for this discrepancy is the long half-life of these myelin structural proteins $(14,15)$. Interestingly, although the number of samples was limited, the $\mathrm{Fl}^{\text {hip}}{ }^{\mathrm{PL}} \mathrm{P}^{\mathrm{lo}}$ lesions did not exhibit significantly lower oligodendrocyte numbers or lipid levels than did control tissues, suggesting the existence of additional demyelinating mechanisms that were independent of oligodendrocyte death or lipid reduction (Figure 7, D and G).

In support of the important role of the Qki-PPAR $\beta-R X R \alpha$ complex in the pathogenesis of PPMS, we found that QKI-5 was reduced specifically in the $\mathrm{Fl}^{\mathrm{lo}} \mathrm{PLP} \mathrm{P}^{\mathrm{hi}}$ group for which myelin lipid reduction is the major pathogenic alteration (Figure 7, D and $\mathrm{H}$ ). We next determined the expression levels of the genes (normalized with the PLP expression level) of 8 Qki-PPAR $\beta$-RXR $\alpha$-associated lipid metabolism pathways, including fatty acid metabolism, fatty acid biosynthesis, biosynthesis of unsaturated fatty acids, fatty acid elongation, fatty acid degradation, PPAR signaling, glycerophospholipid metabolism, and sphingolipid metabolism, from published microarray data (40); a gene set enrichment analysis (GSEA) revealed that the activities of these pathways were significantly downregulated in the MS lesions relative to controls (Figure 7, I and J, and Supplemental Figure 9E). Notably, the levels of $S C D$, SCD5, HACD1, ELOVL1, and ELOVL5, the known Qki-PPAR $\beta-R X-$ $\mathrm{R} \alpha$ target genes that encode key enzymes for fatty acid desaturation and elongation, were all significantly lower in the MS lesions than controls (Figure 7I and Supplemental Figure 9E). Together, our data indicate that disruption of the Qki-PPAR $\beta-R X R \alpha$ complex-regulated lipid metabolism may play an important role in pathological demyelination of subsets of patients with MS.

\section{Discussion}

Myelin has long been considered to be an inert material (14-17), but our studies with $Q k$-iCKO and Ppard-iCKO genetic mouse models reveal that myelin itself, independent of oligodendrocyte survival, is a highly dynamic substance. We found that mature myelin homeostasis requires a continuous Qki-PPAR $\beta$-RXR $\alpha$-dependent biosynthesis of the structural lipid components, particularly unsaturated and elongated fatty acids (Supplemental Figure 9F), probably owing to their high vulnerability to exogenous insults (5). An intriguing observation in our study is that depletion of Qki or PPAR $\beta$ did not affect oligodendrocyte survival yet had detrimental effects on myelin integrity. We postulate that because myelin sheaths are such extraordinarily large extensions of membrane structure, they have a tremendously higher demand for continuous lipid synthesis than 
A

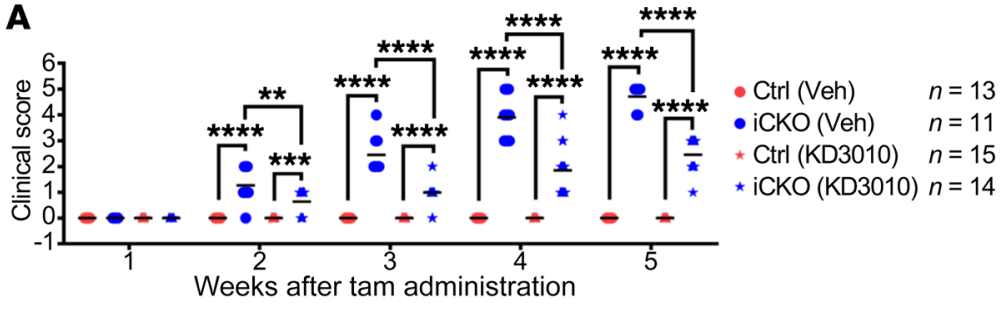

C

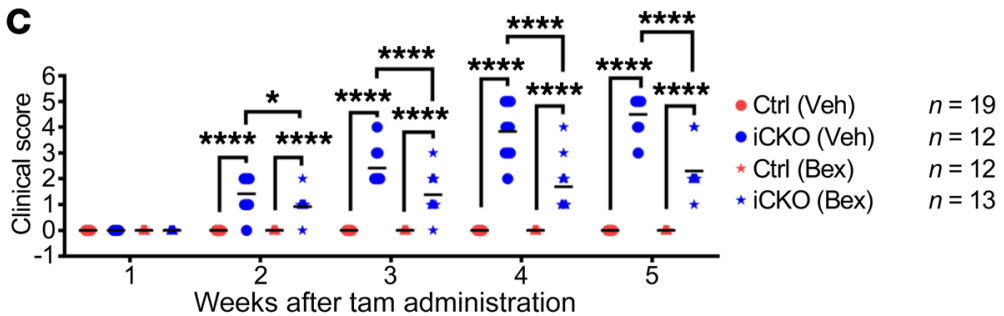

B

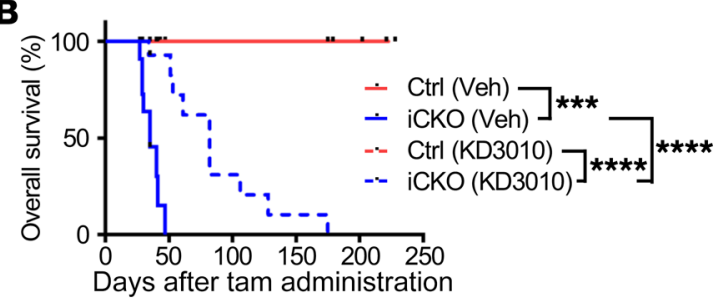

D

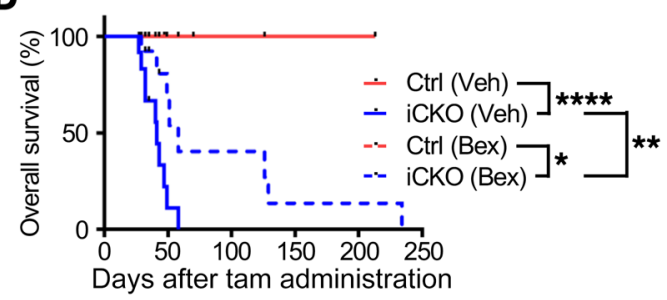

E

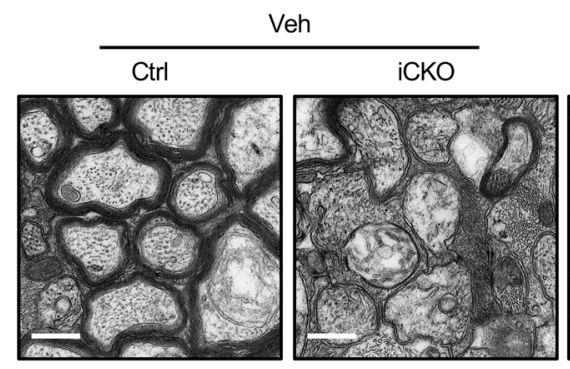

KD3010
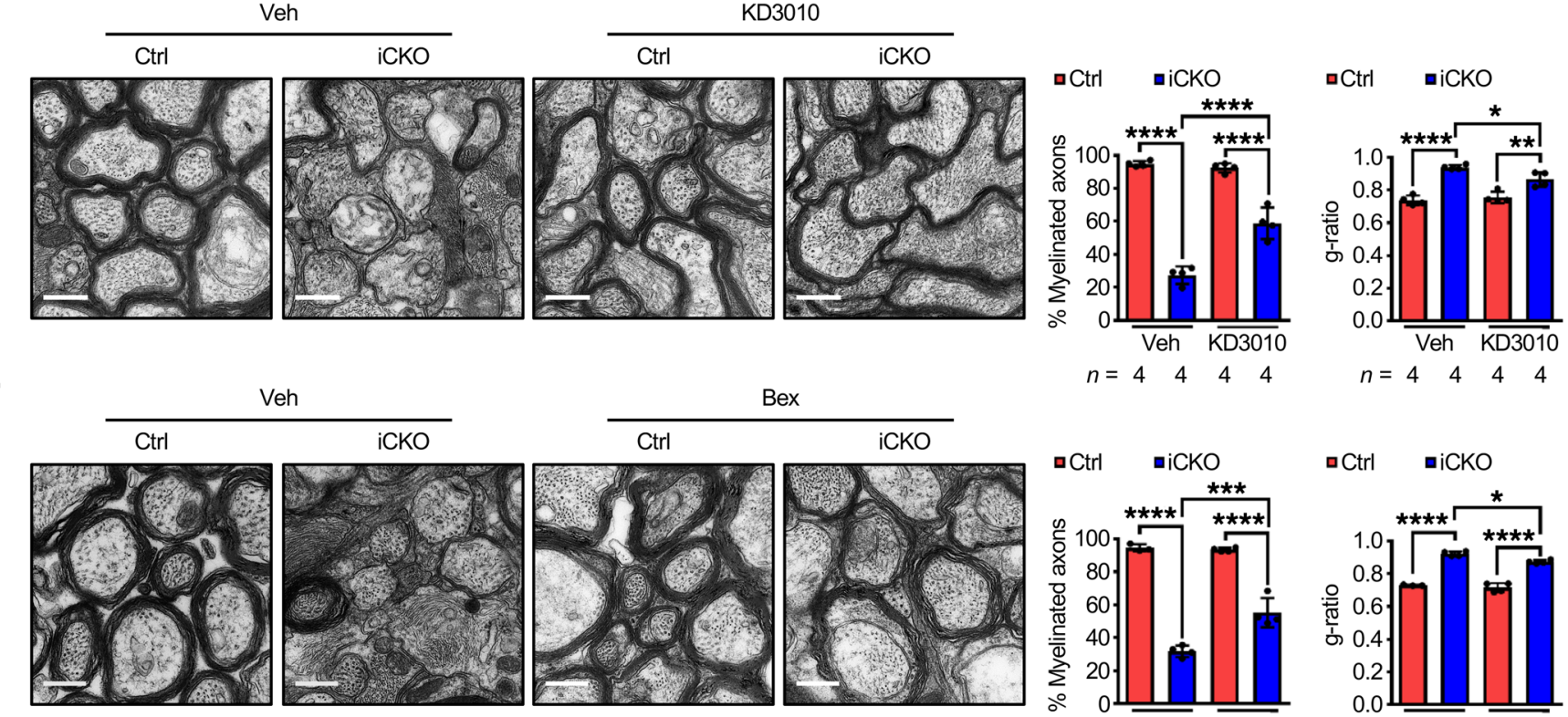

G
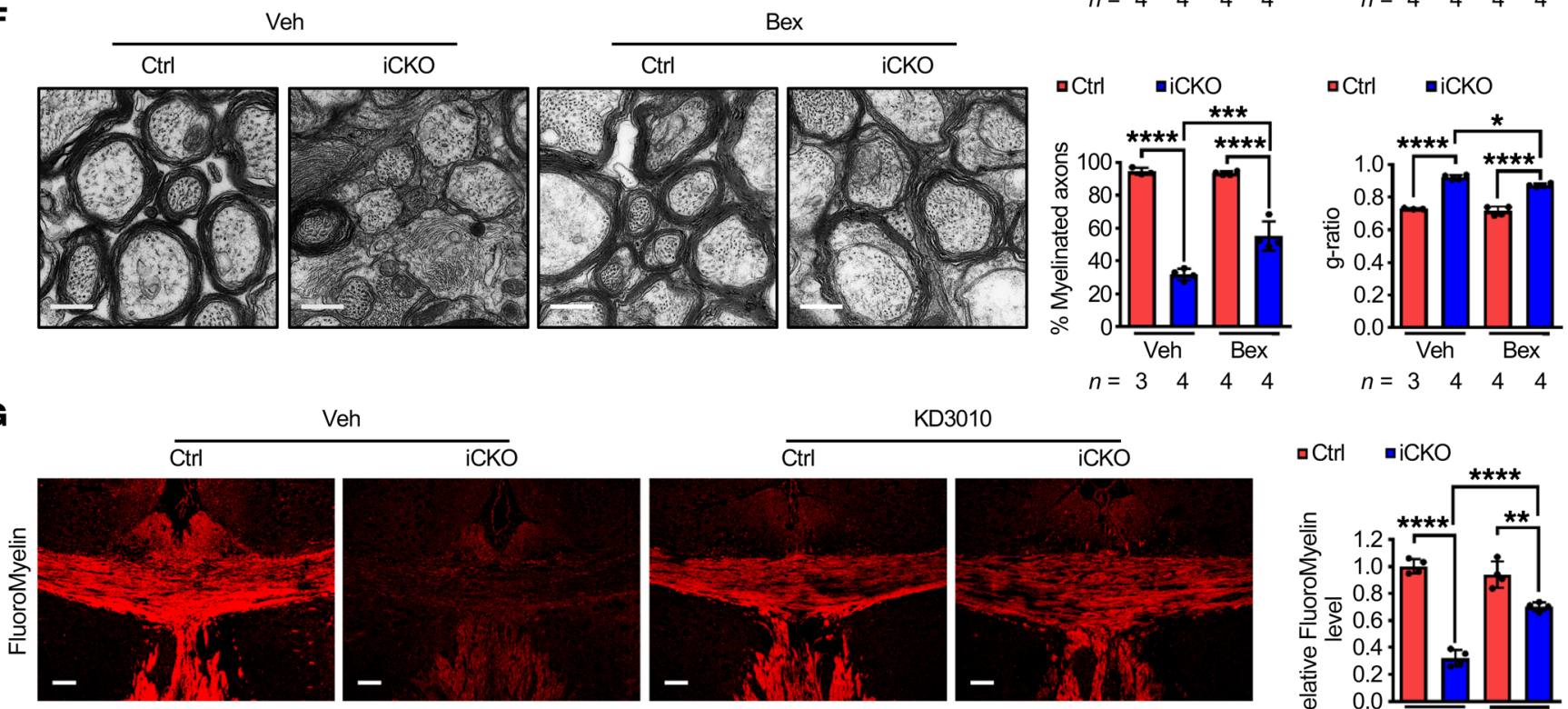

KD3010
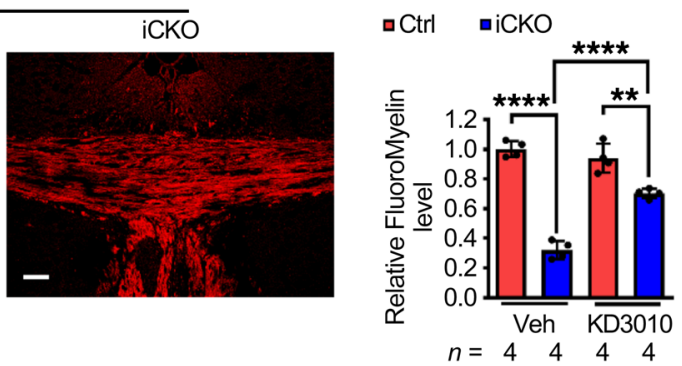

H

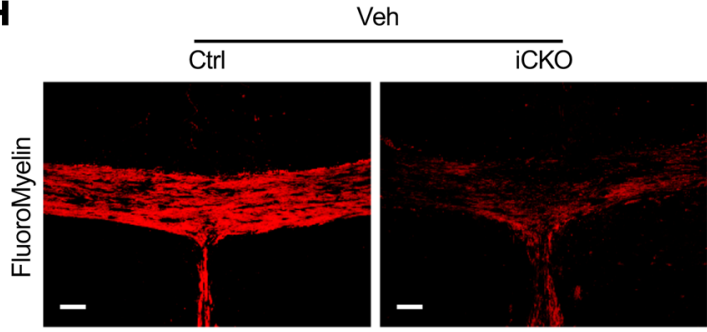

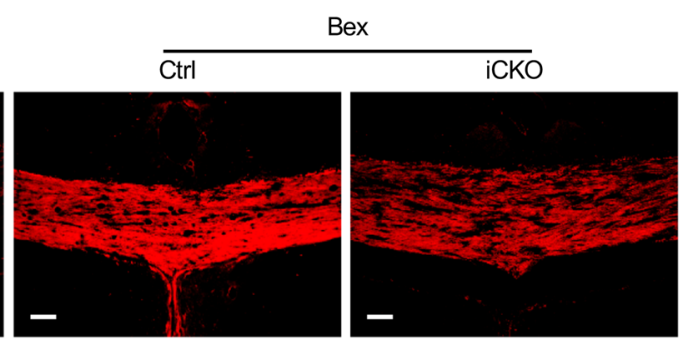


Figure 6. PPAR $\beta$ and RXR agonists alleviate Qki deficiency-induced demyelination. (A-D) The clinical scores and Kaplan-Meier overall survival curves (log-rank test) of $\underline{Q} k$-iCKO mice and controls receiving daily oral administration of KD3010, bexarotene (Bex), or vehicle (Veh). The experimental mouse number is indicated in $\mathbf{A}$ and $\mathbf{C}$. (E and $\mathbf{F}$ ) Representative electron micrographs and quantification of the percentage of myelinated axons and g-ratio of the optic nerves of the experimental mice after 5 weeks of treatment. Scale bars: $500 \mathrm{~nm}$. (G and $\mathbf{H}$ ) Representative images and quantification of FluoroMyelin level in the corpus callosum of the experimental mice after 5 weeks of treatment. Scale bars: $50 \mu \mathrm{m}$. Data are mean $\pm \mathrm{SD} .{ }^{*} P<0.05$; ${ }^{* *} P<0.01 ;{ }^{* *} P<0.001$; ${ }^{* * *} P<0.0001$ by 1 -way ANOVA followed by Bonferroni's post hoc test.

do other cell membranes; therefore, myelin membranes should be particularly sensitive to loss of Qki or PPAR $\beta$. Supporting the notion that Qki-dependent lipid metabolism is not essential for general cell survival, our previous work showed that, consistent with Qki's role as a tumor suppressor (41), Qki depletion in Pten/Trp53-deficient NSCs greatly promoted cell growth and gliomagenesis (29).

Distinct from the widely held dogma that Qki functions primarily as an RNA-binding protein in oligodendrocyte differentiation and myelination (23-28), we uncovered what we believe is a previously unknown function of Qki-5 - as a transcriptional coactivator of the PPAR $\beta$-RXR $\alpha$ complex to regulate fatty acid metabolism during myelin homeostasis. Although previous studies have already shown reduced myelin lipid content in the qkv mouse $(42,43)$, which carries a germline mutation resulting in reduced expression of Qki protein (along with 2 other proteins, Pacrg and Park2) from birth (23), it is worth noting that the reduction in all myelin structural components, including myelin lipids and myelin proteins, is secondary to the impaired oligodendrocyte differentiation and subsequent failure of myelin formation in $\mathrm{qkv}$ mice during development (23-28).

We noticed that the demyelinating phenotypes of Ppard-iCKO mice were not as strong as those of $Q k$-iCKO mice, and the $Q k$-deletion-induced demyelinating phenotype was not completely rescued by the HFD, KD3010, or bexarotene (Figures 4 and 6). There are 3 potential explanations for this. First, although the biosynthesis of unsaturated fatty acids, fatty acid metabolism, and fatty acid elongation (which are all downstream of PPAR $\beta$ signaling) are the pathways most profoundly affected by Qki depletion (Figure 3 and Supplemental Figure 4), we acknowledge the potential contributions of Qki to other PPAR $\beta$-independent pathways. In fact, $34.9 \%(10,525$ of 30,179$)$ of Qki-5 DNA binding sites identified by ChIP-seq did not overlap with PPAR $\beta$ DNA binding sites (Figure $5 \mathrm{G}$ ), suggesting that PPAR $\beta$-independent Qki-5 DNA binding sites might also be involved in demyelination in $Q k$-iCKO mice. Second, in investigating the transcriptional downregulation of the fatty acid metabolism genes, we focused on Qki-5, because we found that Qki-5 was located on chromatin and interacted with the PPAR $\beta$-RXR $\alpha$ complex. However, we cannot rule out the possibility that the cytoplasmic Qki-6 and Qki-7 might also contribute to the demyelinating phenotype of $Q k$-iCKO mice in a PPAR $\beta$-independent manner. Lastly, we found that PPAR $\alpha$ could also interact with Qki-5, although this interaction is not as strong as that between PPAR $\beta$ and Qki-5, and it could be disrupted by sonication (data not shown). Therefore, it is possible that PPAR $\alpha$ could compensate for the loss of PPAR $\beta$ activity in Ppard-iCKO mice. Nevertheless, because no myelin defects have been reported in Ppara-KO mice (44), our data suggested that PPAR $\beta$ is a more important mediator of Qki's function in myelin maintenance than PPAR $\alpha$.

Various studies, by taking advantage of ChIP-seq, showed that between $4 \%$ and $49 \%$ of PPAR $\beta$-binding sites resided in promoter regions $(45-48)$. One explanation for this large variation is that these studies (as well as ours) were performed in different cell types. Of note, other than a 16-bp consensus sequence PPRE, NFIA/NFIX, Rfx, NF1, Sp1, NRF1, and Sox9 motifs were also identified as highly enriched motifs by both our PPAR $\beta$ ChIP and HA ChIP (data not shown), indicating that various cofactors might codetermine where PPAR $\beta$ interacts on the chromatin. In line with our finding, other studies have also found that PPAR $\beta$ cooperated with other transcription factors to specifically regulate subsets of genes, and these factors could be tissue or cell type dependent (46).

The great variability in MS genetics, clinical course, appearance on imaging, pathology, and response to therapy suggests that this chronic demyelinating disorder has multiple etiologies (2). Autoimmune attack (49) and autonomous oligodendrocyte death (3) are thought to be the primary pathogenic events in MS. Contrary to these hypotheses, which both imply a major role for oligodendrocyte death in MS, other studies have noted that 30\%-60\% of early active lesions exhibit no apparent oligodendrocyte death $(6-8)$. Our research found that dysregulation of the myelin lipid metabolism (in both $Q k$-iCKO and Ppard-iCKO mice) is sufficient to drive a demyelinating syndrome without affecting oligodendrocyte viability, indicating that defective lipid metabolism may play an important role in the formation of some MS lesions.

Most disease-modifying therapies that are used to manage relapsing-remitting MS have been found to be ineffective in progressive forms of the disease (50). There is a dire need for interventions that slow or block the chronic, progressive component of MS. Excitingly, the therapeutic benefits of the HFD, KD3010, and bexarotene in $Q k$-iCKO mice raise the possibility that similar approaches would be efficacious in a subset of patients with progressive MS with reduced lipid synthesis in oligodendrocytes and/ or in individuals with leukodystrophies such as diffusely abnormal white matter, in which the myelin lipid level is greatly reduced yet the myelin protein level is relatively unchanged (51).

\section{Methods}

Mice. Mice bearing the Plp-CreER ${ }^{\mathrm{T} 2}$ allele (C57BL/6) (52) and mice bearing the Ppard-loxP allele (C57BL/6) (53) were obtained from The Jackson Laboratory. Mice with the Nestin-CreER ${ }^{\mathrm{T} 2}$ allele (C57BL/6) were provided by Ryoichiro Kageyama (Kyoto University, Kyoto, Japan) (54). Mice with the $Q k$-loxP allele were described previously (29). Mice bearing the Qk-loxP allele were crossed with mice bearing the Plp-CreER ${ }^{\mathrm{T} 2}$ allele, in which the expression of tamoxifen-inducible Cre is under the control of the Plp promoter; the Plp-CreER $R^{\mathrm{T} 2} \mathrm{Qk}^{f / f l}, \mathrm{Plp}-\mathrm{CreER} R^{\mathrm{T} 2} \mathrm{Qk}^{\mathrm{fl} /+}$, or WT mice were generated and used in further experiments. Mice bearing the $Q k-$ loxP allele were crossed with mice bearing the Nestin-CreER ${ }^{\mathrm{T} 2}$ allele, in which the expression of tamoxifen-inducible Cre is under the control of the Nestin promoter, and Nestin-CreER $R^{\mathrm{T} 2} Q k^{f / f l}$ pups were used for NSC isolation. All mouse work was performed under pathogen-free conditions at MD Anderson's animal facility. Mice were fed a standard chow unless otherwise specified, maintained under a 12-hour light/dark cycle, housed in groups, and monitored for signs of ill health every other day. 
A

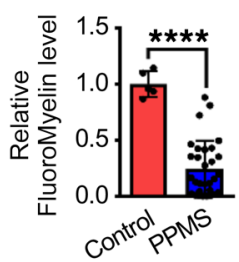

B

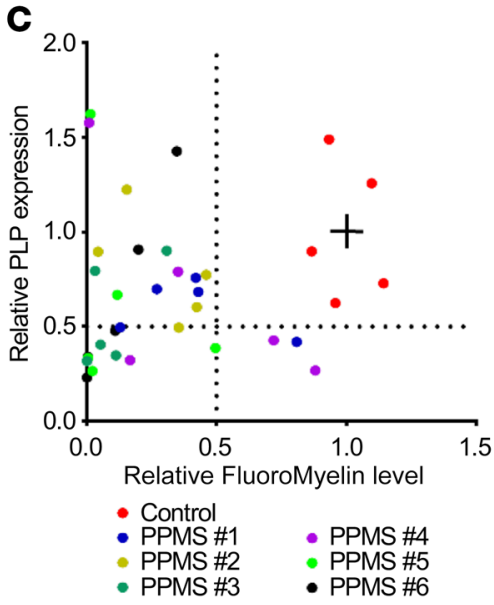

E

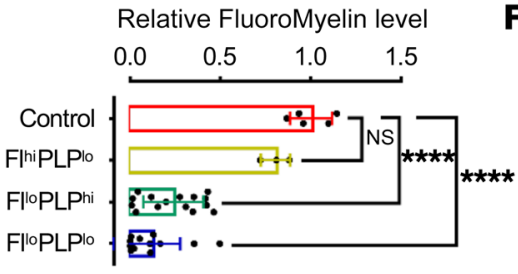

D FluoroMyelin
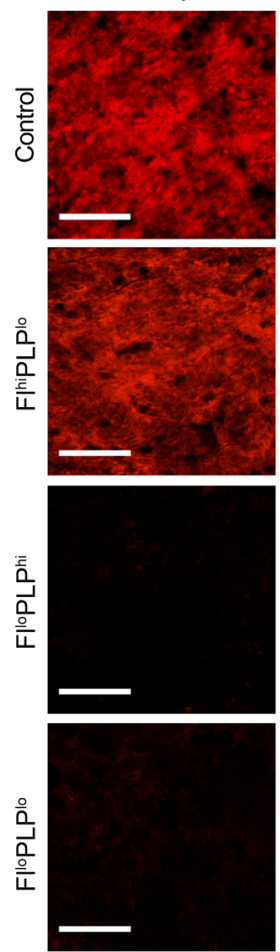

F Relative PLP expression $\begin{array}{lllll}0.0 & 0.5 & 1.0 & 1.5 & 2.0\end{array}$

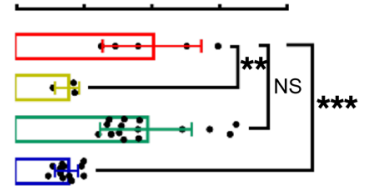

PLP
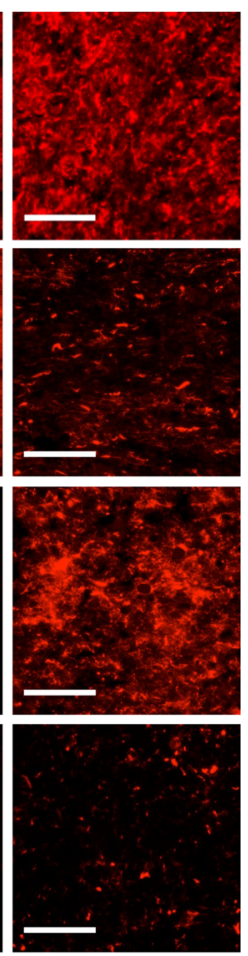

G

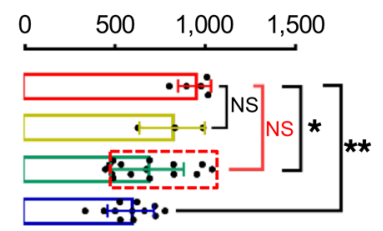

QKI-5
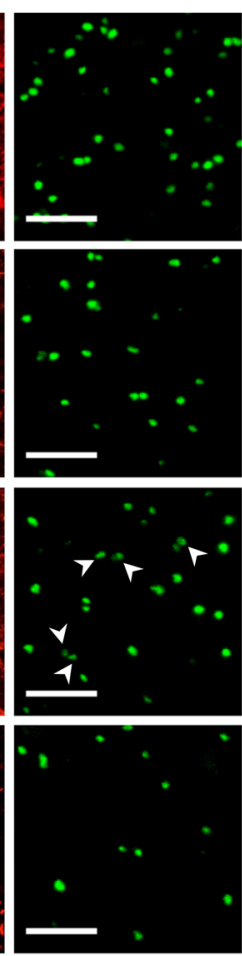

$\longrightarrow$

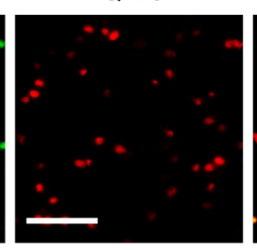

OLIG2/QKI-5
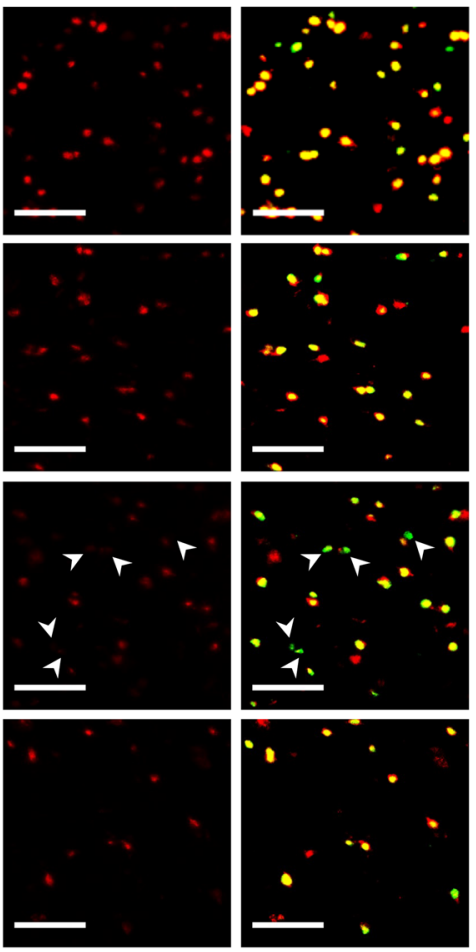

H Relative QKI-5 expression $\begin{array}{llllll}0.7 & 0.8 & 0.9 & 1.0 & 1.1 & 1.2\end{array}$

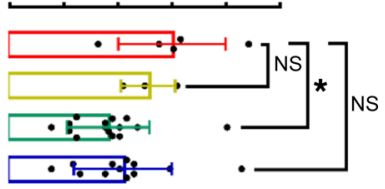

I Biosynthesis of unsaturated fatty acids

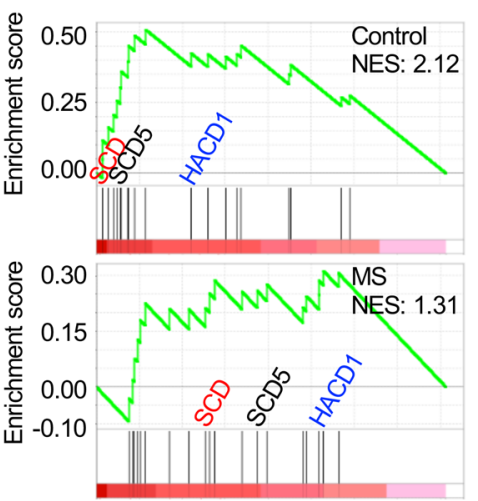

Fatty acid metabolism

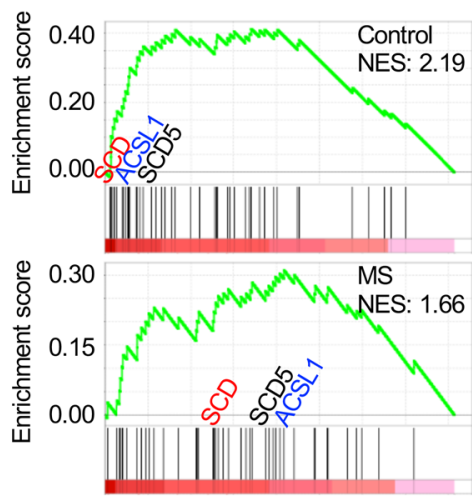

J

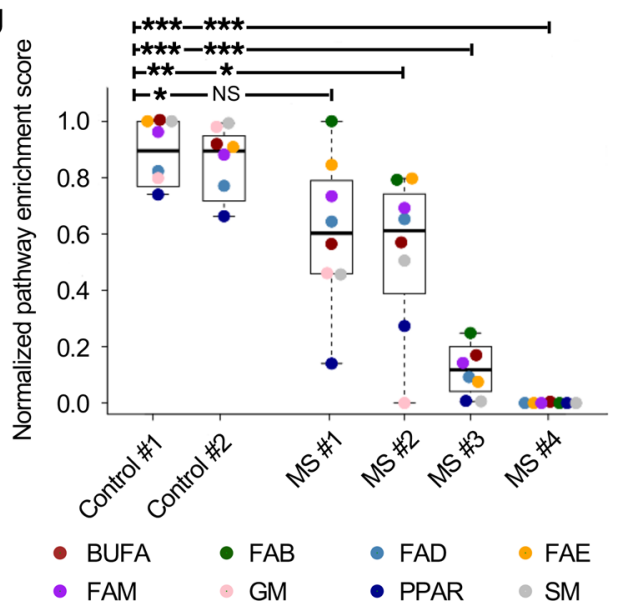

Figure 7. Lipids are more vulnerable than proteins in PPMS lesions. (A and B) Quantification of staining of FluoroMyelin (A) and PLP (B) in the frontal lobes from 5 human brains without neurological disease (control) and 30 lesions from 6 patients with PPMS. (C) Scatter plot of FluoroMyelin intensity versus PLP intensity in the samples in $\mathbf{A}$. The solid lines among red dots represent the average levels of FluoroMyelin and PLP in controls. (D-H) Representative images (D) and quantification of FluoroMyelin level (E), PLP level (F), OLIG2+ cell number (C), and QKI-5 level (H) in controls and 3 subtypes of PPMS lesions, which were divided according to the level of FluoroMyelin and PLP in C. $n=5$ in the control group; $n=3$ in the Fl ${ }^{\text {hipLPlo }}$ group; $n=15$ in the FllopLPhi group; $n=12$ in the $\mathrm{FI}^{\mathrm{lo} P L P^{10}}$ group. Scale bars: $50 \mu \mathrm{m}$. (I and J) Comparison of CSEA of fatty acid metabolism pathways (I) and the NES (J) between MS lesions and the controls. Box plots indicate medians, interquartile values, ranges, and individual pathways. BUFA, biosynthesis of unsaturated fatty acids; FAB, fatty acid biosynthesis; FAD, fatty acid degradation; FAE, fatty acid elongation; FAM, fatty acid metabolism; CM, glycerophospholipid metabolism; SM, sphingolipid metabolism. Data are mean $\pm \mathrm{SD} .{ }^{*} P<0.05 ;{ }^{* *} P<0.01 ;{ }^{* *} P<0.001 ;{ }^{* * *} P<0.0001$ by Student's $t$ test $(\mathbf{A}$ and B), 1-way ANOVA followed by Bonferroni's post hoc test $(\mathbf{E}-\mathbf{H})$, or Wilcoxon's signed-rank test (J). NS, not significant. 
When the Plp-CreER $R^{\mathrm{T} 2} Q k^{f / f l}$ mice were 8 weeks old, $100 \mu \mathrm{L}$ of tamoxifen $(10 \mathrm{mg} / \mathrm{mL}$ in corn oil; Sigma-Aldrich) was injected subcutaneously on 2 consecutive days to induce the deletion of $Q k$ in oligodendrocytes. Littermates of both sexes with the same genetic backgrounds and of the same ages were used for all experiments. For the high fatty acid diet rescue experiment, 8-week-old mice were fed either a normal chow or chow supplemented with $13.68 \%$ SFAs and 14\% MUFAs (58Y1; TestDiet). For the KD3010 rescue experiment, 8-week-old mice were orally administered $50 \mathrm{mg} / \mathrm{kg}$ /day KD3010 or vehicle $(0.5 \%$ carboxymethyl cellulose containing $12.5 \%$ ethanol). For the bexarotene rescue experiment, 8-week-old mice were given $100 \mathrm{mg} / \mathrm{kg} /$ day oral bexarotene (Cayman Chemical) or vehicle (0.5\% carboxymethyl cellulose). For the in vivo Brdu incorporation assay, $50 \mathrm{mg} / \mathrm{kg}$ BrdU (Sigma-Aldrich) in phosphate-buffered saline (PBS) was injected into the peritoneal cavity every 8 hours for 4 days before scarification.

The tamoxifen-injected $Q k$-iCKO mice and controls were monitored daily, and their neurological deficits were assessed weekly using a modified experimental autoimmune encephalomyelitis scoring system to characterize the disease phenotype and severity. In brief, 3 types of neurological deficits, including tremor, hind limb weakness, and paresis, were scored on a scale from 0 to 2 . Tremor was scored as T0 (no tremor), T1 (tremor when the tail was holding), or T2 (tremor when walking or standing). Hind limb weakness was rated as WO (normal), W1 (an angle between the hind leg and paw of around $90^{\circ}$ ), or W2 (an angle between the hind leg and paw of around $180^{\circ}$ ). Hind limb paresis was categorized as PO (normal), P1 (splayed gait and ataxia), or P2 (hind limb paresis or paralysis). The clinical score was the sum of these 3 ratings, ranging from 0 (TOWOPO) to 6 (T2W2P2). When the clinical score reached 5 or 6 , most of the mice showed a hunched posture, immobility, and loss of body weight and were moribund. Thus, they were considered to have reached the clinical endpoint and were euthanized if they were not found dead. Euthanized mice were considered in the analyses in the same way as those that died, with the time of death or euthanasia plotted accordingly on the survival curves.

Mice bearing the Ppard-loxP allele were crossed with mice bearing the Plp-CreER ${ }^{\mathrm{T} 2}$ allele. The 8-week-old Plp-CreER ${ }^{\mathrm{T} 2} \mathrm{Ppard}^{l / f l}$ mice were subcutaneously injected with $100 \mu \mathrm{L}$ of tamoxifen $(10 \mathrm{mg} / \mathrm{mL})$ on 2 consecutive days to induce the deletion of Ppard in oligodendrocytes specifically. To assess motor coordination and balance, the experimental mice were tested on a rotarod apparatus (Harvard Bioscience, Inc.). After 3 days of training, mice were tested in a 2-minute trial on the rotarod apparatus rotating at $20 \mathrm{rpm}$. If a mouse stayed on the rotarod longer than 2 minutes, a time of 120 seconds was recorded. Mice were tested 4 times, with half-hour intervals between each trial. For statistical analyses, a mean latency of falling from the rotarod of 4 trials of each mouse was used.

Human tissue specimens. Fresh-frozen tissue specimens were obtained from the Rocky Mountain MS Center Tissue Bank and stored at $-80^{\circ} \mathrm{C}$ until use. In total, 11 postmortem brain slices were analyzed, including 5 control brain slices from donors without neurological disease and 6 from patients with PPMS. The patients' age at the time of death, sex, postmortem interval, pathological diagnosis, cause of death, disease duration, and disease-modifying therapies are shown in Supplemental Table 3. Lesions were recognized grossly, dissected, and embedded in optimal cutting temperature compound. Twenty-micrometer-thick frozen sections were sliced and stored at $-80^{\circ} \mathrm{C}$. Histological classification of PPMS lesions (5 from each patient) was characterized following the Lassmann/Brück method (39). Briefly, reduction in the intensity of Luxol fast blue stain and/or immunohistochemistry of MBP was used to indicate the demyelinating activity; the presence/absence and distribution of macrophages/microglia (immunohistochemistry of CD68) was used to indicate inflammatory activity (39). The frontal lobe white matter from neurological disease-free human brains was used as control tissue. The intensities of PLP and FluoroMyelin (Invitrogen) were quantified and normalized with the average level of those in control tissue. The levels of FluoroMyelin and PLP that were lower than $50 \%$ of the average level of those in control tissues were defined as $\mathrm{Fl}^{\mathrm{lo}}$ and $\mathrm{PLP}^{\mathrm{lo}}$, respectively; all of the lesions were grouped into 3 groups based on their myelin lipid and protein levels: Fl ${ }^{\text {lo }}$ L $P^{\text {hi }}, \mathrm{Fl}^{\text {hi }} \mathrm{PLP}^{\mathrm{lo}}$, and Fl ${ }^{\mathrm{lo}} \mathrm{PLP} \mathrm{P}^{\mathrm{lo}}$.

NSC isolation and oligodendrocyte differentiation. Mouse NSCs were isolated using neural tissue dissociation kits according to the manufacturer's instructions (Miltenyi Biotec). In brief, whole Nestin-CreER ${ }^{\mathrm{T} 2}$ $Q k^{f / f l}$ mouse forebrains from postnatal day 1 pups (both sexes) were dissociated enzymatically and the single-cell suspension was cultured in NeuroCult Basal Medium (Stemcell Technologies), supplemented with $20 \mathrm{ng} / \mathrm{mL}$ epidermal growth factor (ProteinTech), $10 \mathrm{ng} / \mathrm{mL}$ basic fibroblast growth factor (ProteinTech), NeuroCult Proliferation Supplement (Stemcell Technologies), 50 units/mL penicillin G, and $50 \mu \mathrm{g} /$ $\mathrm{mL}$ streptomycin in a humidified $37^{\circ} \mathrm{C}$ incubator with an atmosphere containing $5 \% \mathrm{CO}_{2}$. The NSCs were exposed to $100 \mathrm{nM} 4$-hydroxytamoxifen (Sigma-Aldrich) twice at an interval of 2 days to knockout Qk. To induce oligodendrocyte differentiation, NSCs were cultured on dishes coated with poly-L-ornithine (Sigma-Aldrich) and laminin (Thermo Fisher Scientific) and maintained in neurobasal medium (Thermo Fisher Scientific) containing B-27 (Thermo Fisher Scientific), 2 mM GlutaMAX-I (Thermo Fisher Scientific), $100 \mathrm{ng} / \mathrm{mL}$ insulinlike growth factor 1 (IGF-1) (Peprotech), $50 \mathrm{ng} / \mathrm{mL} \mathrm{3,3',5-triiodo-L-}$ thyronine (T3) (Cayman Chemical), 50 units/mL penicillin $\mathrm{G}$, and 50 $\mu \mathrm{g} / \mathrm{mL}$ streptomycin. IGF-1 and T3 were added daily, and the differentiation medium was replaced with fresh medium every other day.

Primary oligodendrocyte isolation. Tamoxifen was administered subcutaneously to 8-week-old $Q k$-iCKO mice and controls on 2 consecutive days. Four days later, brains were dissociated enzymatically according to the instructions of the Adult Brain Dissociation Kit (Stemcell Technologies). After the removal of debris and red blood cells, the single-cell suspension was incubated with anti-O4 microbeads, followed by magnetic separation. The $\mathrm{O}^{+}$oligodendrocytes and the $\mathrm{O}^{-}$- flow-through were collected separately for further RTqPCR validation. The $\mathrm{O}^{+}$oligodendrocytes from $Q k$-iCKO mice and controls were used for RNA sequencing.

Tissue preparation and immunofluorescence. Mice were anesthetized with isoflurane and perfused transcardially with $4 \%$ formaldehyde. Their brains, spinal cords, and optic nerves were removed, postfixed in formalin at room temperature for 2 days, and embedded in paraffin. Five-micrometer-thick paraffin-embedded sections were deparaffinized and subjected to heat-induced antigen retrieval in citrate buffer (Poly Scientific R\&D Corp.). The sections were blocked with $10 \%$ horse serum and incubated with primary antibodies overnight at $4^{\circ} \mathrm{C}$ in the blocking solution. The following antibodies were used for staining, according to their manufacturer's directions: antiOlig2 (catalog AB9610), anti-Olig2 (catalog MABN50), anti-aspartoacylase (anti-ASPA) (catalog ABN1698), and anti-MBP (QD-9; catalog AB5864) were from Merck Millipore; anti-IBA1 (catalog ab107159), 
anti-Brdu (catalog ab6326), anti-PLP (catalog ab105784), anti-PPAR $\beta$ (catalog ab137724), and anti-CD68 (catalog ab955) were from Abcam; anti-MAG (catalog 9043), anti-PDGFR $\alpha$ (catalog 3174), and anticleaved caspase 3 (catalog 9661) were from Cell Signaling Technology; anti-QKI (catalog SAB5201536) was from Sigma-Aldrich; anti-GFAP (catalog 556330) was from BD Biosciences; anti-GSTpi (catalog 311) was from MBL International; anti-MBP (catalog SMI-94R) was from Covance; anti-QKI-5 (catalog IHC-00574) was from Bethyl Laboratories; anti-QKI-6 (catalog 75-190) and anti-AnkG (catalog MABN466) were from NeuroMab; anti-QKI-7 was from a rabbit immunized with a short synthetic peptide (KGGPIEPSGVLEWIEMPVMPDISAH); antiCD3 (catalog A0452) was from DAKO; and anti-Caspr was a gift from Matthew N. Rasband (Baylor College of Medicine, Houston, Texas, USA). After 3 washes with Tris-buffered saline containing 0.1\% Tween 20 , the sections were incubated with species-appropriate secondary antibodies conjugated with Alexa Fluor dyes for 1 hour. To mount the stained sections, mounting medium with DAPI (Vector Laboratories) was used. All stained sections were visualized using a Leica DMi8 microscope, and images were obtained using a Leica DFC345 FX digital monochrome camera.

Frozen sections were thawed at room temperature for 30 minutes and fixed in ice-cold formalin for 10 minutes. After being rehydrated with cold PBS, sections were blocked with $10 \%$ horse serum and incubated with primary antibodies and secondary antibodies, as described above. For FluoroMyelin staining, the rehydrated slides were stained directly for 20 minutes at room temperature.

Strong autofluorescence was stimulated in the human brain sections by multiple wavelength channels of the microscope; it was removed in 2 ways. For staining of OLIG2, autofluorescence was removed using the TrueBlack Lipofuscin Autofluorescence Quencher, according to the manufacturer's instructions (Biotium); the fluorescence images were obtained normally. For FluoroMyelin, PLP, MBP, and MAG, immunofluorescent staining was performed normally using secondary antibodies that were specifically stimulated by the red wavelength channel. The sections were imaged using both the red channel and the green channel, and signals that appeared in both channels were considered autofluorescent noise. Using this information, we devised an algorithm for enhancing the signal-to-noise ratio of the images. The intensities of the green-filtered image were mean-centered. This mean was subtracted from the red image to remove strong background noise signals. The green image was then converted to a binary (black-and-white) format by setting the threshold at the intensity returned by the Greythresh function in Matlab software. The Matlab Regionprops function was used to obtain bounding boxes for all spots observed in the black-and-white-converted green image. The corresponding spots in the red-filtered image were substituted with the mean intensity of the red image. Pixels within the bounding boxes of the red image, corresponding to all spots identified in the green image, were then subjected to Gaussian filtering to blur these regions and thereby minimize noise.

Electron microscopy. Tamoxifen was administered to 8-week-old Qk-iCKO mice and controls. After 1, 2, and 5 weeks, the mice were anesthetized with isoflurane and transcardially perfused with $2 \%$ paraformaldehyde. The corpus callosum, optic nerves, and spinal cords were removed and postfixed in a solution containing $2 \%$ paraformaldehyde and $3 \%$ glutaraldehyde in PBS at $4^{\circ} \mathrm{C}$. Fixed samples were processed at the High Resolution Electron Microscopy Facility at
MD Anderson. Digital images were obtained using the AMT Imaging System (Advanced Microscopy Techniques Corp.).

Electrophysiology. Compound action potential (CAP) recordings in optic nerves were performed as described elsewhere $(55,56)$. Briefly, optic nerves were dissected and immediately placed in a continuously perfused, oxygenated, and temperature-regulated $\left(25^{\circ} \mathrm{C}\right)$ chamber containing standard Locke's solution (154 mM NaCl, $5.6 \mathrm{mM} \mathrm{KCl,} 2$ $\mathrm{mM} \mathrm{CaCl}_{2}, 5 \mathrm{mM}$ D-glucose, and $10 \mathrm{mM}$ HEPES [pH 7.4]). The ends of the nerve were drawn orthodromically into suction electrodes, with the retinal side in the stimulating electrode and the chasmic side in the recording electrode. Nerves were then stimulated and the responses to a depolarizing current were amplified, digitized, recorded, and analyzed using Molecular Devices Axon pClamp, MultiClamp, and Clampfit software. Nerve conduction velocity was calculated by dividing the length of the optic nerve by the time from stimulation to the peak of the CAP.

Complex lipidomic analyses. Tamoxifen was administered to 8-week-old $Q k$-iCKO mice and controls. Each spinal cord was collected, weighed, and lipids were extracted for further mass spectrometric analyses to yield data from each mouse ( $n=5$ mice/group). Lipidomic analyses were performed by Metabolon, Inc. Lipids were extracted from the spinal cords using dichloromethane and methanol in a modified Bligh-Dyer extraction procedure, in the presence of internal standards, with the lower organic phase used for analysis. The extracts were concentrated under nitrogen and reconstituted in 0.25 $\mathrm{mL}$ of dichloromethane/methanol (50:50) containing $10 \mathrm{mM}$ ammonium acetate. The extracts were placed in vials for ion-mobility spectrometry analyses, performed on a SelexION-equipped SCIEX 5500 QTRAP mass spectrometer with both positive- and negative-mode electrospray. Each sample was analyzed twice, with the ion-mobility spectrometry-mass spectrometry conditions optimized for the lipid classes monitored in each analysis. The 5500 QTRAP mass spectrometer was operated in multiple reaction monitoring mode to monitor the transitions for over 1,100 lipids from up to 14 lipid classes, including neutral lipids (cholesteryl ester, triacylglycerol, diacylglycerol, and free fatty acid), phospholipids (phosphatidylcholine, phosphatidylethanolamine, phosphatidylinositol, lysophosphatidylcholine, and lysophosphatidylethanolamine), and sphingolipids (sphingomyelin, ceramide, hexosylceramide, lactosylceramide, and dihydroceramide). Individual lipid species were quantified based on the ratio of the signal intensity for the target compound to the signal intensity for an assigned internal standard of known concentration. Lipid class concentrations ( $\mu \mathrm{mol} / \mathrm{g}$ spinal cord tissue) were calculated by summing the quantities of all molecular species within a class, and fatty acid compositions were determined by calculating the proportion of individual fatty acids within each class.

Stable cells. The coding DNA sequence region of WT or mutant Qki-5 with an HA tag at the C-terminus was subcloned into the lentiviral vector pInducer20 (57), and the lentiviruses packaged in HEK293T cells were used to infect Qki-depleted NSCs. After selection with G418 (Thermo Fisher Scientific), the surviving cells were treated with doxycycline (Sigma-Aldrich) to induce the expression of HA-Qki-5 (WT or mutant). Similarly, the coding DNA sequence region of Ppard gene was subcloned into pInducer20, and doxycycline-inducible PPAR $\beta$-expressing stable cells were generated in NSCs.

Dual-luciferase reporter assay. HEK293T cells were seeded in 24-well plates. A mixture of a PPAR-responsive firefly luciferase report- 
er, a constitutively expressing Renilla element, a pInducer20-PPAR $\beta$ construct, and a pInducer20-Qki-5 construct (or the empty vector) was cotransfected. Forty-eight hours after cells were transfected and treated with doxycycline, they were lysed, and luciferase activity was measured using a dual-luciferase reporter assay system (Promega), following the manufacturer's instructions. Transfection efficiency was normalized to Renilla luciferase activity.

RNA sequencing and pathway enrichment analyses. Total RNA was isolated as described in Supplemental Methods and treated with DNase (RNase-free, Qiagen). Sequencing was performed by the Illumina HiSeq/MiSeq sequencing service at the Sequencing and Microarray Facility at MD Anderson. Raw reads were aligned with the mouse reference genome ( $\mathrm{mm} 10$ version) using the TopHat program, allowing 2 mismatches. The DEseq 2 package for $\mathrm{R}$ was used to perform normalization and create a gene expression matrix. All RNA sequencing data were analyzed from at least 3 biological replicates. The fold-change threshold at which genes were considered to be differentially expressed was set at 1.2-fold with $P$ less than 0.05. Significantly affected pathways were analyzed using Advaita Bio's iPathwayGuide (http://www.advaitabio.com/ipathwayguide). In addition, GSEA was carried out as described previously to identify functional enrichment profiles (58).

Data availability. The RNA sequencing data and ChIP sequencing data reported in this study have been deposited in the NCBI's Gene Expression Omnibus (GEO) under ID codes GSE84134, GSE106468, GSE125581, and GSE126577.

Statistics. The numerical results are presented as mean \pm standard deviation (SD) and were compared using a 2-tailed Student's $t$ test (unpaired), 2-way ANOVA with Holm-Sidak multiple-comparisons test, or 1-way ANOVA followed by Bonferroni's post hoc test, as indicated. Animal survival durations were analyzed using the log-rank test. Statistical analyses were conducted using GraphPad Prism 6. The numbers of animals or cells used to perform the experiments are indicated in the figure legends. For immunoblotting and RT-qPCR, representative data from multiple independent replicates are shown. No statistical methods were used to predetermine sample size. $P$ values of less than 0.05 were considered to be statistically significant in all cases. There were no randomization or blinding events during the experiments.

All other methods are detailed in Supplemental Methods.
Study approval. All mouse work was conducted in accordance with protocols approved by the Institutional Animal Care and Use Committee of The University of Texas MD Anderson Cancer Center (Houston, Texas). Informed consent and ethical approval for the use of human samples were collected by the Rocky Mountain MS Center Tissue Bank. MD Anderson provided guidelines for human tissue study procedures.

\section{Author contributions}

$\mathrm{JH}$ and XZ designed the study and analyzed the data. XZ conducted the experiments. $\mathrm{CH}$ and FL performed ChIP and the bioinformatics analysis for ChIP-seq. JR and QW performed the GSEA. JR, CD, TS, and LY assisted in sample preparation for RNA-seq. SRS and MNR performed electrophysiology. DZ, YW, FZ, and YC performed the bioinformatics analysis for RNA-seq. CRC performed mouse genotyping. VR and AUKR designed the algorithm for removing autofluorescence. $\mathrm{HZ}, \mathrm{ABH}$, and $\mathrm{BMS}$ helped in the analyses of human tissue specimens. JH and $\mathrm{XZ}$ wrote the manuscript.

\section{Acknowledgments}

We thank Shan Jiang, Kun Zhao, and Yanping Cao for mouse husbandry and care and all members of the Hu laboratory for helpful discussions. We thank Kenneth Dunner, Jr. for performing electron microscopy studies. We thank John R. Corboy and Caroline Miller for providing the human brain specimens from the Rocky Mountain Multiple Sclerosis Center Tissue Bank, University of Colorado. We thank Albert R. La Spada for providing KD3010 as the positive control for our own KD3010 synthesis. We also thank David M. Wildrick and the Department of Scientific Publications of MD Anderson for providing editorial assistance. This investigation was supported in part by a grant from the National Multiple Sclerosis Society and a Cancer Prevention and Research Institute of Texas grant (RP120348 and RP170002). JH is supported by the University of Texas Rising STARs Award, the Sidney Kimmel Scholar Award, the Sontag Foundation Distinguished Scientist Award, NIH grant R37CA214800, and the Brockman Foundation.

Address correspondence to: Jian Hu, 1881 East Road Unit 1906, Houston, Texas 77054, USA. Phone: 713.794.5238; Email: JHu3@ mdanderson.org.
1. Armati PJ, Mathey EK. The Biology of Oligodendrocytes. Cambridge, UK: Cambridge University Press; 2010.

2. Lassmann H, Brück W, Lucchinetti C. Heterogeneity of multiple sclerosis pathogenesis: implications for diagnosis and therapy. Trends Mol Med. 2001;7(3):115-121.

3. Traka M, Podojil JR, McCarthy DP, Miller SD, Popko B. Oligodendrocyte death results in immune-mediated CNS demyelination. Nat Neurosci. 2016;19(1):65-74.

4. Locatelli G, et al. Primary oligodendrocyte death does not elicit anti-CNS immunity. Nat Neurosci. 2012;15(4):543-550.

5. Bartzokis G. Age-related myelin breakdown: a developmental model of cognitive decline and Alzheimer's disease. Neurobiol Aging. 2004;25(1):5-18; author reply 49.

6. Brück W, et al. Oligodendrocytes in the early course of multiple sclerosis. Ann Neurol. 1994;35(1):65-73.

7. Ozawa K, et al. Patterns of oligodendroglia pathology in multiple sclerosis. Brain. 1994;117(pt 6):1311-1322.

8. Lucchinetti C, Brück W, Parisi J, Scheithauer $\mathrm{B}$, Rodriguez M, Lassmann H. A quantitative analysis of oligodendrocytes in multiple sclerosis lesions. A study of 113 cases. Brain. 1999;122(pt 12):2279-2295.

9. Yeung MS, et al. Dynamics of oligodendrocyte generation and myelination in the human brain. Cell. 2014;159(4):766-774.

10. Peters A. The effects of normal aging on myelinated nerve fibers in monkey central nervous system. Front Neuroanat. 2009;3:11.

11. Bartzokis G, Beckson M, Lu PH, Nuechterlein $\mathrm{KH}$, Edwards N, Mintz J. Age-related changes in frontal and temporal lobe volumes in men: a magnetic resonance imaging study. Arch Gen Psychiatry. 2001;58(5):461-465.

12. Tripathi RB, Jackiewicz M, McKenzie IA Kougioumtzidou E, Grist M, Richardson WD. Remarkable stability of myelinating oligodendrocytes in mice. Cell Rep. 2017;21(2):316-323.

13. Chrast R, Saher G, Nave KA, Verheijen MH. Lipid metabolism in myelinating glial cells: lessons from human inherited disorders and mouse models. JLipid Res. 2011;52(3):419-434.

14. Price JC, Guan S, Burlingame A, Prusiner SB, Ghaemmaghami S. Analysis of proteome dynamics in the mouse brain. Proc Natl Acad Sci US A. 2010;107(32):14508-14513

15. Toyama BH, et al. Identification of long-lived proteins reveals exceptional stability of essential cellular structures. Cell. 2013;154(5):971-982.

16. Smith ME. The turnover of myelin in the adult rat. Biochim Biophys Acta. 1968;164(2):285-293. 
17. Ando S, Tanaka Y, Toyoda Y, Kon K. Turnover of myelin lipids in aging brain. Neurochem Res. 2003;28(1):5-13.

18. Vaswani KK, Ledeen RW. Long-chain acyl-coenzyme A synthetase in rat brain myelin. J Neurosci Res. 1987;17(1):65-70.

19. Koul O, Chou KH, Jungalwala FB. UDP-galactose-ceramide galactosyltransferase in rat brain myelin subfractions during development. Biochem J. 1980;186(3):959-969.

20. Koenning M, et al. Myelin gene regulatory factor is required for maintenance of myelin and mature oligodendrocyte identity in the adult CNS. J Neurosci. 2012;32(36):12528-12542.

21. Ishii A, Furusho M, Dupree JL, Bansal R. Role of ERK1/2 MAPK signaling in the maintenance of myelin and axonal integrity in the adult CNS. J Neurosci. 2014;34(48):16031-16045.

22. Hussien Y, Podojil JR, Robinson AP, Lee AS, Miller $\mathrm{SD}$, Popko B. ER chaperone BiP/GRP78 is required for myelinating cell survival and provides protection during experimental autoimmune encephalomyelitis. J Neurosci. 2015;35(48):15921-15933.

23. Chénard CA, Richard S. New implications for the QUAKING RNA binding protein in human disease. J Neurosci Res. 2008;86(2):233-242.

24. Sidman RL, Dickie MM, Appel SH. Mutant mice (Quaking and Jimpy) with deficient myelination in the central nervous system. Science. 1964;144(3616):309-311.

25. Darbelli L, Vogel G, Almazan G, Richard S. Quaking regulates neurofascin 155 expression for myelin and axoglial junction maintenance. J Neurosci. 2016;36(14):4106-4120.

26. Zhao L, Tian D, Xia M, Macklin WB, Feng Y. Rescuing $\mathrm{qkV}$ dysmyelination by a single isoform of the selective RNA-binding protein QKI. J Neurosci. 2006;26(44):11278-11286.

27. Larocque D, et al. Nuclear retention of MBP mRNAs in the quaking viable mice. Neuron. 2002;36(5):815-829.

28. Li Z, Zhang Y, Li D, Feng Y. Destabilization and mislocalization of myelin basic protein mRNAs in quaking dysmyelination lacking the QKI RNA-binding proteins. J Neurosci. 2000;20(13):4944-4953.

29. Shingu T, et al. Qki deficiency maintains stemness of glioma stem cells in suboptimal environment by downregulating endolysosomal degradation. Nat Genet. 2017;49(1):75-86.

30. Matsuo A, Akiguchi I, Lee GC, McGeer EG, McGeer PL, Kimura J. Myelin degeneration in multiple system atrophy detected by unique antibodies. Am J Pathol.1998;153(3):735-744.
31. Weil MT, et al. Loss of myelin basic protein function triggers myelin breakdown in models of demyelinating diseases. Cell Rep. 2016;16(2):314-322.

32. Monsma PC, Brown A. FluoroMyelin ${ }^{\mathrm{TM}}$ Red is a bright, photostable and non-toxic fluorescent stain for live imaging of myelin. J Neurosci Methods. 2012;209(2):344-350.

33. Stubbs CD, Smith AD. The modification of mammalian membrane polyunsaturated fatty acid composition in relation to membrane fluidity and function. Biochim Biophys Acta. 1984;779(1):89-137.

34. Grygiel-Górniak B. Peroxisome proliferator-activated receptors and their ligands: nutritional and clinical implications--a review. Nutr J. 2014;13:17.

35. Peters JM, et al. Growth, adipose, brain, and skin alterations resulting from targeted disruption of the mouse peroxisome proliferator-activated receptor beta(delta). Mol Cell Biol. 2000;20(14):5119-5128.

36. Dickey AS, et al. PPAR- $\delta$ is repressed in Huntington's disease, is required for normal neuronal function and can be targeted therapeutically. Nat Med. 2016;22(1):37-45.

37. Cramer PE, et al. ApoE-directed therapeutics rapidly clear $\beta$-amyloid and reverse deficits in AD mouse models. Science. 2012;335(6075):1503-1506.

38. Kipp M, Nyamoya S, Hochstrasser T, Amor S. Multiple sclerosis animal models: a clinical and histopathological perspective. Brain Pathol. 2017;27(2):123-137.

39. Kuhlmann T, Ludwin S, Prat A, Antel J, Brück W, Lassmann H. An updated histological classification system for multiple sclerosis lesions. Acta Neuropathol. 2017;133(1):13-24.

40. Han MH, et al. Janus-like opposing roles of CD47 in autoimmune brain inflammation in humans and mice. JExp Med. 2012;209(7):1325-1334

41. Darbelli L, Richard S. Emerging functions of the Quaking RNA-binding proteins and link to human diseases. Wiley Interdiscip Rev RNA. 2016;7(3):399-412.

42. Baumann NA, Jacque CM, Pollet SA, Harpin ML. Fatty acid and lipid composition of the brain of a myelin deficient mutant, the "quaking" mouse. Eur J Biochem. 1968;4(3):340-344.

43. Baumann N, Bourre JM, Jacque C, Harpin ML. Lipid composition of Quaking mouse myelin: comparison with normal mouse myelin in the adult and during development. J Neurochem . 1973;20(3):753-759.

44. Lee SS, et al. Targeted disruption of the alpha isoform of the peroxisome proliferator-activated receptor gene in mice results in abolishment of the pleiotropic effects of peroxisome prolifera- tors. Mol Cell Biol. 1995;15(6):3012-3022.

45. Adhikary T, et al. Genomewide analyses define different modes of transcriptional regulation by peroxisome proliferator-activated receptor- $\beta / \delta$

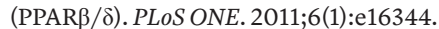

46. Khozoie $\mathrm{C}$, et al. Analysis of the peroxisome proliferator-activated receptor- $\beta / \delta(\operatorname{PPAR} \beta / \delta)$ cistrome reveals novel co-regulatory role of ATF4. BMC Genomics. 2012;13:665.

47. Adhikary T, et al. The transcriptional PPAR $\beta / \delta$ network in human macrophages defines a unique agonist-induced activation state. Nucleic Acids Res. 2015;43(10):5033-5051.

48. Fan W, et al. PPAR $\delta$ promotes running endurance by preserving glucose. Cell Metab. 2017;25(5):1186-1193.e4

49. McFarland HF, Martin R. Multiple sclerosis: a complicated picture of autoimmunity. Nat Immunol. 2007;8(9):913-919.

50. Segal BM, Stüve O. Primary progressive multiple sclerosis--why we are failing. Lancet. 2016;387(10023):1032-1034.

51. Laule C, et al. Diffusely abnormal white matter in multiple sclerosis: further histologic studies provide evidence for a primary lipid abnormality with neurodegeneration. J Neuropathol Exp Neurol. 2013;72(1):42-52.

52. Doerflinger NH, Macklin WB, Popko B. Inducible site-specific recombination in myelinating cells. Genesis. 2003;35(1):63-72.

53. Barak Y, et al. Effects of peroxisome proliferator-activated receptor delta on placentation, adiposity, and colorectal cancer. Proc Natl Acad Sci US A. 2002;99(1):303-308.

54. Imayoshi I, et al. Roles of continuous neurogenesis in the structural and functional integrity of the adult forebrain. Nat Neurosci. 2008;11(10):1153-1161.

55. Rasband MN, Peles E, Trimmer JS, Levinson SR, Lux SE, Shrager P. Dependence of nodal sodium channel clustering on paranodal axog lial contact in the developing CNS. J Neurosci. 1999;19(17):7516-7528.

56. Susuki K, et al. Gangliosides contribute to stability of paranodal junctions and ion channel clusters in myelinated nerve fibers. Glia. 2007;55(7):746-757.

57. Meerbrey KL, et al. The pINDUCER lentiviral toolkit for inducible RNA interference in vitro and in vivo. Proc Natl Acad Sci US A. 2011;108(9):3665-3670.

58. Subramanian A, et al. Gene set enrichment analysis: a knowledge-based approach for interpreting genome-wide expression profiles. Proc Natl Acad Sci U S A. 2005;102(43):15545-15550. 